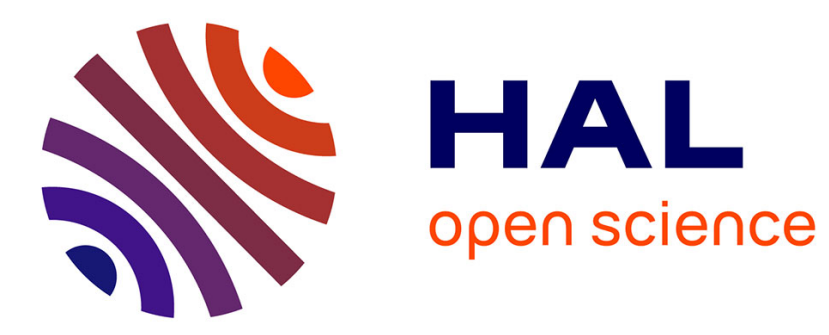

\title{
On the connection of isolated branches of a bifurcation diagram: the truss arch system
}

\author{
Nathalie M. M. Cousin-Rittemard, Isabelle Gruais
}

\section{To cite this version:}

Nathalie M. M. Cousin-Rittemard, Isabelle Gruais. On the connection of isolated branches of a bifurcation diagram: the truss arch system. Dynamical System: an international journal, 2009, 24 (3), pp.315-341. 10.1080/14689360802691037 . hal-00833032

\section{HAL Id: hal-00833032 https://hal.science/hal-00833032}

Submitted on 11 Jun 2013

HAL is a multi-disciplinary open access archive for the deposit and dissemination of scientific research documents, whether they are published or not. The documents may come from teaching and research institutions in France or abroad, or from public or private research centers.
L'archive ouverte pluridisciplinaire HAL, est destinée au dépôt et à la diffusion de documents scientifiques de niveau recherche, publiés ou non, émanant des établissements d'enseignement et de recherche français ou étrangers, des laboratoires publics ou privés. 
Dynamical System : an international journal, 24 (2009), no. 3, 315-341.

\title{
On the connection of isolated branches of a bifurcation diagram: the truss arch system.
}

\author{
N.M.M. Cousin-Rittemard and I. Gruais \\ I.R.M.A.R, Université de Rennes1, Campus de Beaulieu,35042 Rennes Cedex, France. \\ Corresponding author. Email: nathalie.rittemard@univ-rennes1.fr
}

\begin{abstract}
One of the important basic issues of bifurcation theory is the determination of the set of the fixed points of non-linear evolution equations as a function of its parameters. The branching of branches of solutions rarely occurs in real applications for which imperfections tend to distort these sharp transitions. Furthermore, bifurcation theory may a priori indicate that there are disjoint branches of solutions. In the present work, the truss arch system is considered and described. The bifurcation diagram is carried out numerically. It is shown that the truss arch system is a simple example of coexistence of disjoint branches. Moreover, it is shown that the emergence of the subcritical bifurcations of the non-shallow configuration is the result of the connection of these disjoint branches. The analytic solutions are derived and the connection of the branches is studied.
\end{abstract}

Keywords:

\section{Introduction}

One of the important basic issues of bifurcation theory is the determination of the set of the equilibrium points of non-linear evolution equations as a function of its parameters. As a first approach, the bifurcation theory may be seen as the study of the branching of the solutions of the corresponding set of equations:

$$
\mathbf{A}(\mathbf{u}, \mu)=\mathbf{0}
$$

where $\mathbf{u} \in \mathbb{R}^{n}$ and $\mu \in \mathbb{R}^{\mathbf{p}}$ are the vectors of the unknowns and parameters respectively, $\mathbf{A}: \mathbb{R}^{n} \times \mathbb{R}^{p} \rightarrow \mathbb{R}^{n}$ is a nonlinear operator with $n \leq+\infty$. From this point of view, one may see the branching as a perfect bifurcation. Infinitesimal changes in a control parameter of such a nonlinear system may yield large and sudden changes of the behaviour of the system. For example, the snap buckling of the elastic rods or shells are well known examples of jump phenomena (See e.g. [1]). We refer to [2] for an up-to-date state of the art. 
Nevertheless, the branching of solutions rarely occurs in real applications for which imperfections tend to distort these sharp transitions [3]. A typical example is the imperfect pitchfork bifurcation occurring in the wind-driven ocean flows $[4,5]$. Furthermore, bifurcation theory may a priori indicate that there are disjoint branches of solutions [6]. It seems relevant to distinguish between the branching or local bifurcations which can be entirely analysed through the stability properties of equilibria as parameters cross through critical thresholds and the global bifurcations which often occur when larger invariant sets collide with each other or with equilibria of the system. The latter cannot be detected purely by a local stability analysis of the fixed points. Examined in a larger parameter space, the catastrophe theory reveals that such bifurcation points tend to occur as part of well-defined qualitative geometrical structures [7]. The most complete set of the fixed points is thus desirable.

In the present paper, the truss arch system is considered and described in section $\S 2$. The bifurcation diagram carried out numerically $[10,9,8]$ is described in $\S 3$. It is shown that the truss arch system is a simple example of coexistence of disjoint branches. Moreover, it is shown that the emergence of the subcritical bifurcations of the non-shallow configuration is the result of the connection of these disjoint branches. In section $\S 4$, the analytic solutions of the equilibria of the truss arch system are derived while in $\S 5$ the connection of the branches is modelized and analysed.

\section{Formulation}

Let us denote $E$ and $A$ the Young moduli and the area of the sections of the elastic rods of the truss arch system. Let $\left(x_{i}, y_{i}\right)$ for $i=1,2,3$, be the coordinates of the left and right hinge supports and of the upper point before loading $F=0$. We denote by $(x, y)$ the coordinates of the point 3 for a given vertical load $|F|>0$. Without any loss of generality, we set: $x_{3}=y_{3}=0$ and we note: $x_{2}=a, x_{1}=-a, y_{1}=y_{2}=h$. According to dimensional analysis (See e.g. [11]), we define the dimensionless unknowns and parameters:

$$
\mathbf{U}(X \equiv x / h, \quad Y \equiv y / h), \quad \Lambda(t \equiv a / h, f \equiv E A F / E A) \quad \text { and } \quad r=t^{-1} .
$$

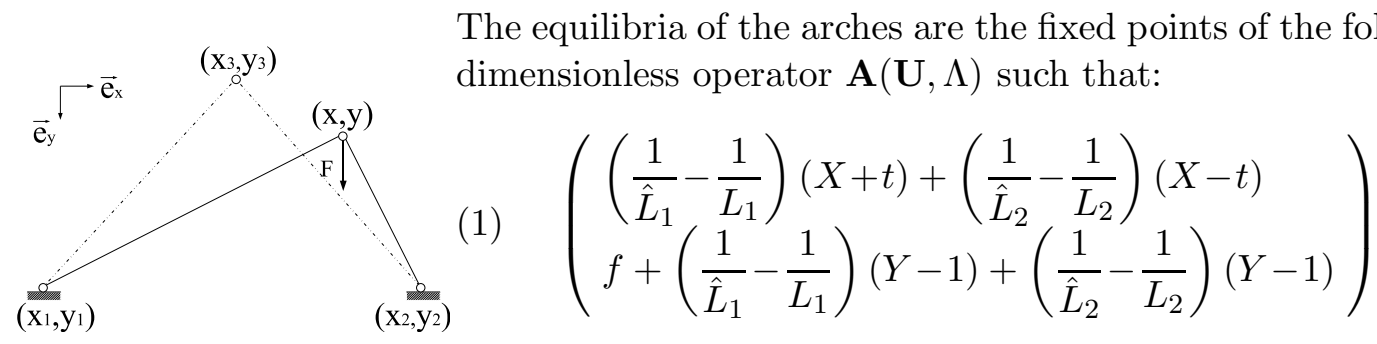

where the dimensionless lengths of the rods at rest and for a given vertical load $f$ respectively are: 


$$
\begin{array}{r}
L=L_{1}=L_{2}=\sqrt{t^{2}+1}, \\
\hat{L}_{1}=\sqrt{(X+t)^{2}+(Y-1)^{2}}, \\
\hat{L}_{2}=\sqrt{(X-t)^{2}+(Y-1)^{2}} .
\end{array}
$$

The system is conservative and the potential $V$ is such that:

$$
\forall K \in \mathbb{R}, V(U, \boldsymbol{\Lambda})=\sum_{i=1}^{2}\left(\frac{\hat{L}_{i}^{2}}{2 L_{i}}-\hat{L}_{i}\right)-f Y+K .
$$

Given $\left(\mathbf{U}_{0}, \Lambda_{0}\right)$ a fixed point of a branch of the operator $\mathbf{A}$, let $\delta \mathbf{U}$ be a perturbation with respect to $\mathbf{U}$. The potential $V\left(\mathbf{U}_{0}+\delta \mathbf{U}, \Lambda_{0}\right)$ is such that:

$$
V\left(\mathbf{U}_{0}+\delta \mathbf{U}, \Lambda_{0}\right) \simeq V\left(\mathbf{U}_{0}, \Lambda_{0}\right)-\frac{1}{2} \delta \mathbf{U}^{t} \mathbf{D}_{\mathbf{U}} \mathbf{A}\left(\mathbf{U}_{0}, \Lambda_{0}\right) \delta \mathbf{U} .
$$

Let $\left(\mathbf{v}_{i}\right)_{i=1,2}$ be a eigenvectors basis of the symmetric jacobian matrix $\mathbf{D}_{\mathbf{U}} \mathbf{A}$. For each $\delta \mathbf{U}=\varepsilon \mathbf{v}_{i}$, it follows :

$$
V\left(\mathbf{U}_{0}+\delta \mathbf{U}, \Lambda_{0}\right) \simeq V\left(\mathbf{U}_{0}, \Lambda_{0}\right)-\frac{1}{2} \varepsilon^{2} \sigma_{i} \mathbf{v}_{\mathbf{i}}{ }^{t} \mathbf{v}_{\mathbf{i}}
$$

where $\sigma_{i}$ is the eigenvalue corresponding to the eigenvector $\mathbf{v}_{i}$. Hence, an equilibrium is unstable if one of the eigenvalues of the jacobian matrix $\mathbf{D}_{\mathbf{U}} \mathbf{A}$ is positive.

\section{Bifurcation Diagrams}

The behavior of truss arches depends on the aspect ratio $r$. The bifurcation diagram of shallow and non-shallow truss arches is numerically computed $[10,9,8]$ and studied. In order to carry out the set of the fixed points as comprehensively as possible, a unified computational approach is used. Starting from a known fixed point, a branch is carried out with Newton-Raphson algorithm with continuation (See e.g. [12, 13, 14]). The disjoint branches are reached using residue continuation [10, 9].

The leading eigenvalues associated with the bifurcations and loss of stability are computed using QR algorithm [15] (See e.g. [16]) as a stable (resp. unstable) state correspond to negative (resp. positive) value of the eigenvalue of the jacobian matrix or, equivalently, a minimum (resp. maximum) of the energy (See §2). In the bifurcation diagrams, the solid and the dashed lines denote the stable and unstable parts of a branch respectively. For the latter case, only one real mode is stable. A critical value of a parameter is labeled with the corresponding bifurcation name. For example, $f_{b 1}$ is the critical load at the bifurcation $\mathrm{b} 1$.

This paragraph shows that the set of the equilibria of the truss arch system is composed of two subsets: the snap-through and the conchoidal solutions. Below the critical 
threshold $r_{r s}$, the two subsets are disjoint as illustrated in $§ 3.1$. The two subsets collide and connect at the threshold as illustrated in Figure 2 in $§ 3.2$. Above the threshold, the corresponding connection leads to the creation of four subcritical bifurcations and the split and recombination of both conchoidal branches into a inner and an outer snap with rotation toward or around the hinge support as briefly described $\S 3.3$.

\subsection{Shallow truss arches}

For shallow configuration, there is not one branch (see e.g. [1, 17, 9]) but three disjoint branches which connect for non-shallow case. For example, Figure 1 depicts the bifurcation diagram for an aspect ratio equal to one.

Branch \#1, along which $X=0$, is the well known catastrophic snap-through phenomenon described as it follows. Starting from rest $(f=0)$ on Branch \#1, the rods are compressed as a function of a vertical load $(f>0)$. At the critical load $f_{\mathrm{b} 1}$, the saddlenode point b1 is eventually reached. The corresponding equilibrium is half-stable. The system snaps down towards the stable point c. From the equilibrium c, the decrease of the load yields to the critical value $f_{\mathrm{b} 2}$. Again, from the saddle-node b2, the system jumps up towards the stable equilibrium d. One highlights that the two saddle-node bifurcations b1 and $\mathrm{b} 2$ lead to a hysteretic behavior as a function of the load $f$.

Disjoint Branches \#423-2 and, symmetrically, \#423-1 are unstable. We named these branches in accordance with the split in three parts (4,2 and 3) that will emerge from connection in non-shallow configuration (See 3.2, 3.3 and 5). Furthermore, we labeled " 1 " the left branch that is for $X<0$ and symmetrically "-2" the right branch that is for $X>0$. Both disjoint Branches straddle the horizontal line between the hinge supports: $Y=1$. The apparent "cusp" points $(X= \pm t Y=1)$ are the positions of the hinge supports for which the two rods are horizontal and either the left or right one is infinitely compressed. At the fold points $(X= \pm L, Y=1)$, the compression of one horizontal rod counterbalances the traction of the other.

\subsection{Connection}

Figure 2 depicts the evolution of Branch \#423-2 as a function of the aspect ratio $r$ within the range $[0.7,2.5]$. As the aspect ratio increases, the curve \#423-2 (symmetrically \#423-

1) becomes closer to Branch \#1 $(X=0)$ as the abcissa of the fold point equal to $L=\sqrt{1+t^{2}}$ tends towards one. It is a part of a continuously deformed conchoid that straddles the horizontal line $(Y=1)$. Eventually, for $r_{r s}$, disjoint Branch \#423-2 (and symmetrically \#423-1) collapses on Branch \#1 throught two cusps. The connection of Branches \#423-2 and \#423-1 give rise to the three unstable branches \#4, \#2 and \#3 and four subcritical bifurcations, which will be described in the next paragraph. As a matter of fact, Branch \#2 is the result of the connection of the collapsed parts of Branches \#423-1 and \#423-2 turning round the hinge supports. Branch \#3 (resp. \#4) is the result of the connection of the collapsed parts of Branches \#423-1 and \#423-2 for which $f>0$ (resp. $f<0$ ) turning towards the hinge supports. 


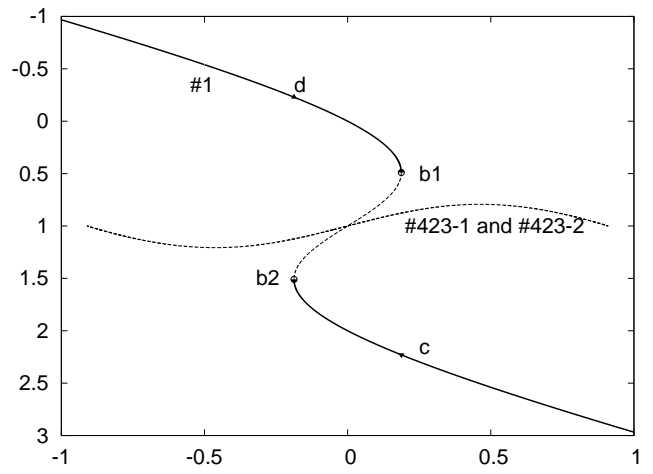

(a) $Y$ versus $f$

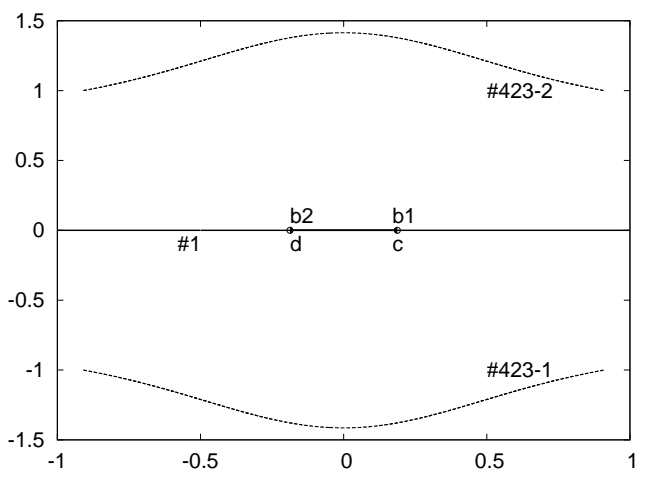

(c) $X$ versus $f$

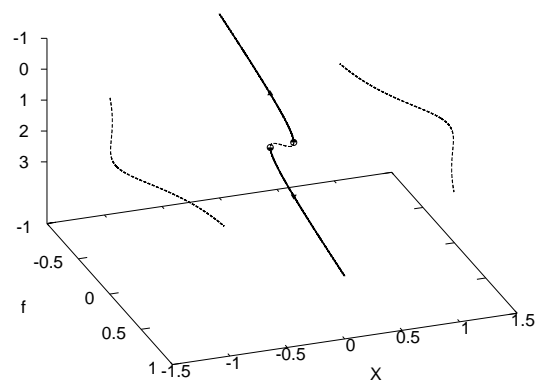

(b) $Y$ versus $f$ and $X$

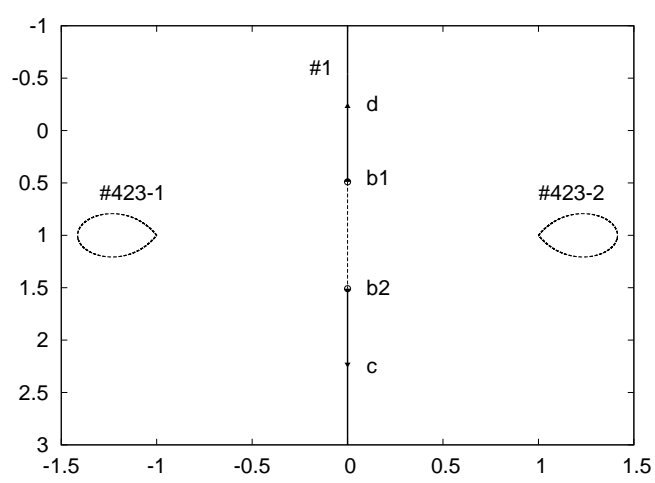

(d) $Y(f)$ versus $X(f)$

Figure 1: Shallow truss arches for $r=1$ : in (a) and (c), $Y$ and $X$ are plotted versus the load $f$, respectively while in (d) the parametric curve $(Y(f), X(f))$ is shown. The three graphs are the left, top and front points of view of the three dimensionnal graph (b) respectively. A solid and dashed linestyle denote the stable and unstable parts of the branches respectively. For the latter case, only one real mode is stable. 


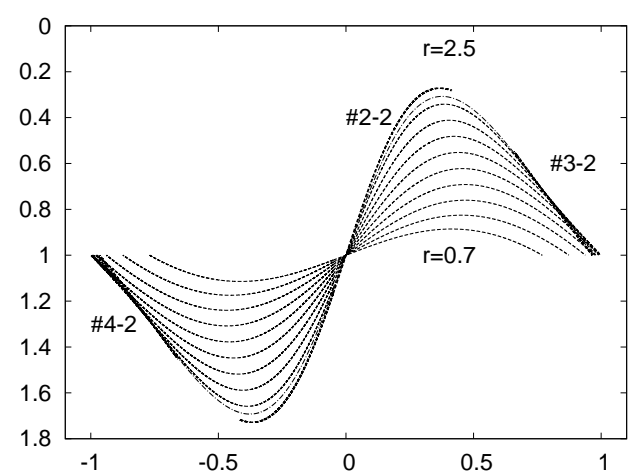

(a) $Y$ versus $f$

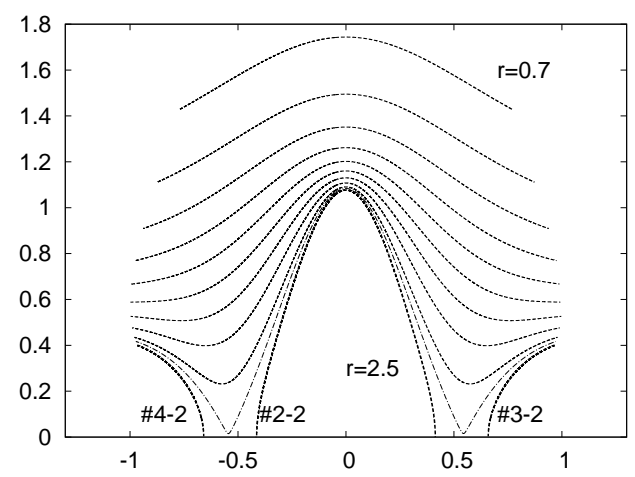

(c) $X$ versus $f$

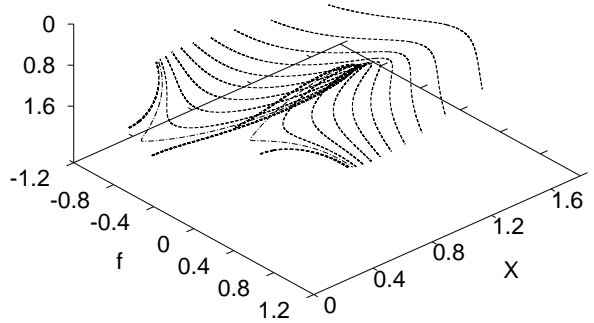

(b) $Y$ versus $f$ and $X$

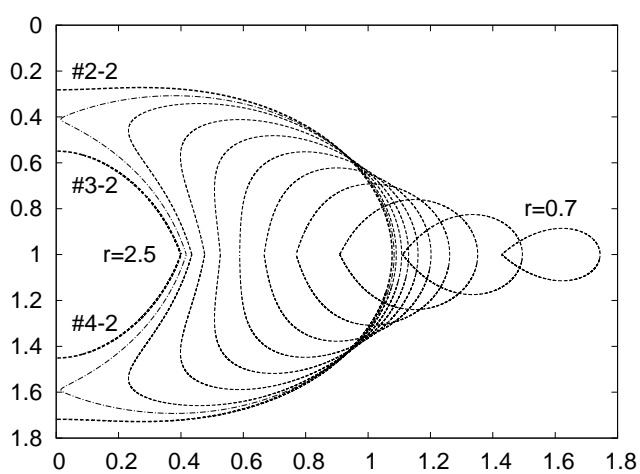

(d) $Y(f)$ versus $X(f)$

Figure 2: Shallow truss arches evolution of Branch \#423-2 for $r \in[0.7,2.5]$ : in (a) and (c), $Y$ and $X$ are plotted versus the load $f$, respectively while in (d) the parametric curve $(Y(f), X(f))$ is shown. The three graphs are the left, top and front points of view of the three dimensionnal graph (b) respectively. The increment of the aspect ratio between two dashed curves is 0.2 . For emphasis, bold dashed curve is used for $r=2.5$. The dashed dotted linestyle is used for the critical aspect ratio $r \sim 2.4 \equiv r_{r s}$. 


\subsection{Non-shallow truss arches}

We first consider $r=2.4$ in the vicinity of the critical aspect ratio (See Figure 3 ). There are the four branches \#1, \#2, \#3 and \#4 and the six bifurcations: r1, s1 and b1 for the positive loads and $\mathrm{r} 2$, s2 and b2 for the negatives ones. Branch \#1 is stable except between the subcritical bifurcations $\mathrm{r} 1-\mathrm{s} 1, \mathrm{r} 2 \mathrm{-s} 2$ and between the saddle node bifurcations b1-b2.

Firstly, the perfectly vertical snap-through can not be observed if one loads from rest. Indeed, before the saddle-node bifurcation b1, Branch \#1 is partly unstable between $\mathrm{r} 1$ and s1. The parametric diagram 3(d) shows that the subcritical Branch \#2 is a rotation. The blowing-up behavior induced could be described as it follows. One loads the system from rest $(f=0)$. At the critical load $f_{\mathrm{r} 1}$, the subcritical point $\mathrm{r} 1$ is eventually reached. The corresponding equilibrium is half-stable. While turning round the hinge supports, the system snaps shortly up and then down, towards the stable point c. From the equilibrium $\mathrm{c}$, the decrease of the load yields the critical value $f_{\mathrm{r} 2}$. Again, from the subcritical pitchfork $\mathrm{r} 2$, the system jumps shortly down and then up to the stable equilibrium $\mathrm{f}$ while turning round the hinge supports. As the vertical snap-through, the so-called outer snap-through is an hysteresis as a function of the load $f$.

Secondly, if one increases or decreases the load starting from a value in the range $\left[f_{\mathrm{s} 1}, f_{\mathrm{b} 1}\right]$, the hysteretic cycle of the outer snap-through eventually occurs after a perfectly vertical or inner snap-through towards the hinge supports respectively.

Both vertical and inner snap-through before the outer hysteresis coexist in the range $r \in[2.4,2.7]$ as soon as the subcritical pitchfork s1 occurs before b1 in this range. Increasing the aspect ratio from $r_{r s}$, the s1 bifurcation tends towards b1 and the limit is $r=2.7$ is a catastrophic cusp point $[18,7]$. For higher values, both subcritical bifurcations s1

and s2 are between the saddle node bifurcations b1 and b2 and tend to each other. The corresponding physical behavior is the outer hysteresis alone as it is described hereafter.

For an aspect ratio equals to five units, Figure 4 depicts $Y$ and $X$ versus the load $f$ and the parametric diagram $Y(f)$ versus $X(f)$. The double dotted lines denote the unstable parts of the branch for which the two real modes are stable. There are the four branches \#1, \#2, \#3 and \#4 and six bifurcations: r1, b1 and s1 for $f>0$ and r2, b2 and s2 for $f<0$. As previously described, the perfectly vertical snap-through would not be observed loading from rest. The outer-snap through occurs. Nevertheless, neither the vertical nor inner snap-through are physically realized. Along branch \#1, the subcritical pitchfork bifurcation s1 occurs after b1. The fold point b1 and the subcritical point s1 induce successively one mode and two modes to be stable. Hence, there is no longer a range of stability after r1. The asymptotic solution [8] highlights the behavior of non-shallow truss arch system. 


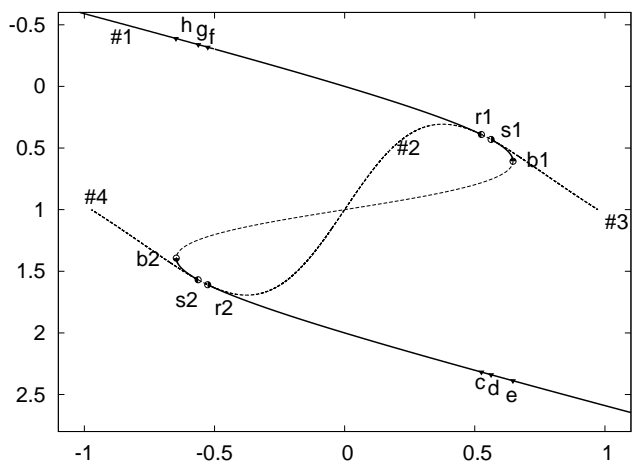

(a) $Y$ versus $f$

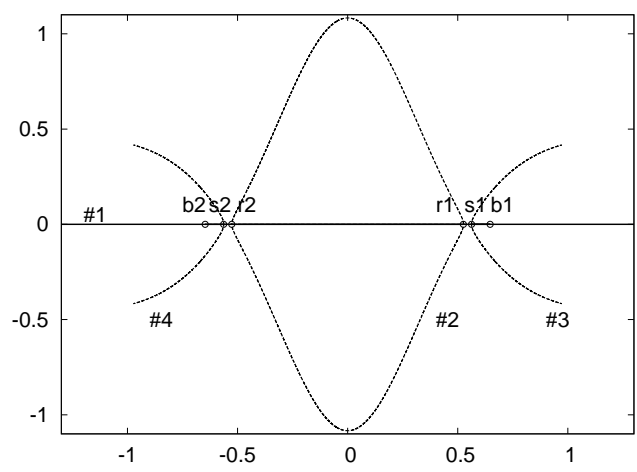

(c) $X$ versus $f$

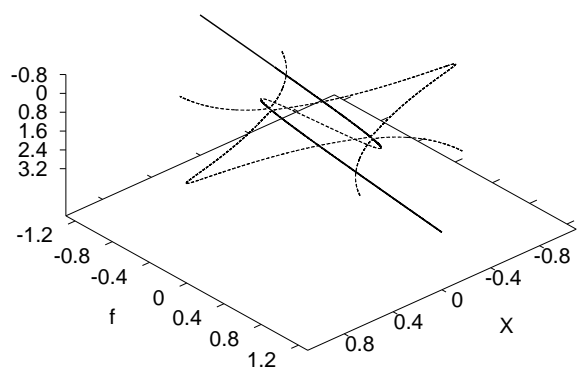

(b) $Y$ versus $f$ and $X$

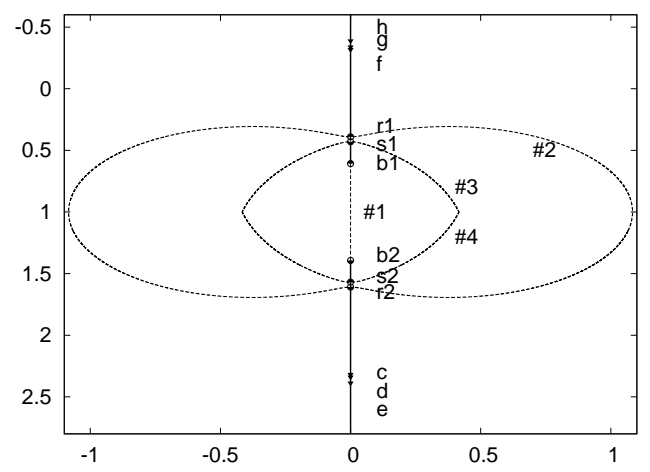

(d) $Y(f)$ versus $X(f)$

Figure 3: Non-shallow truss arches for $r=2.4$ : in (a) and (c), $Y$ and $X$ are plotted versus the load $f$, respectively while in $(\mathrm{d})$ the parametric curve $(Y(f), X(f))$ is shown. The three graphs are the left, top and front points of view of the three dimensionnal graph (b) respectively. A solid and dashed linestyle denote the stable and unstable parts of the branches. For the latter case, only one real mode is stable. 


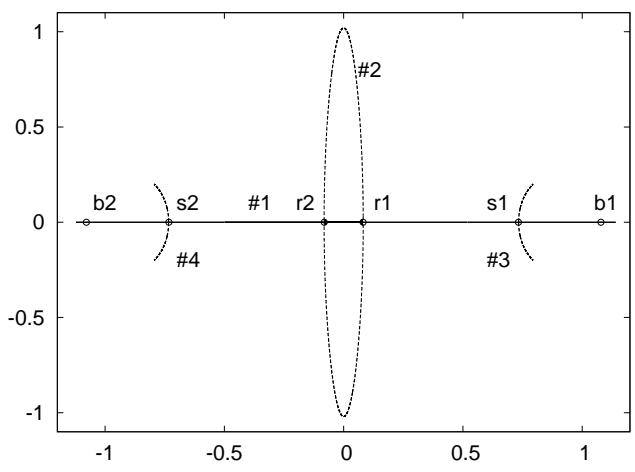

(a) $X$ versus $f$

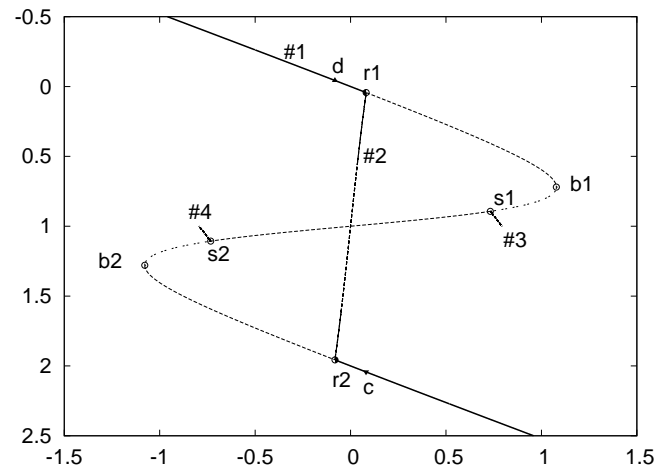

(b) $Y$ versus $f$

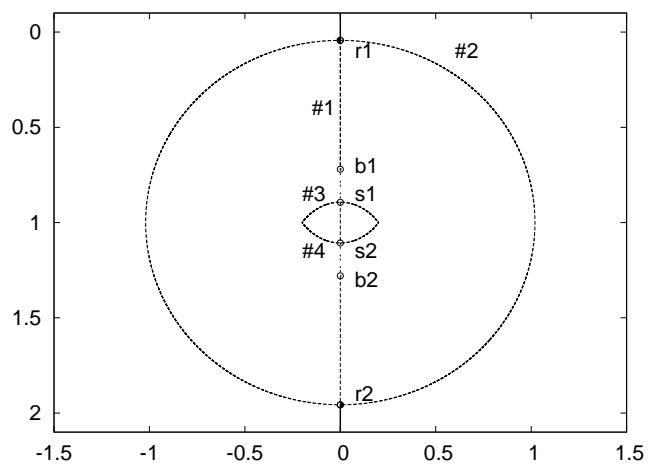

(c) $Y(f)$ versus $X(f)$

Figure 4: Non shallow truss arches for $r=5$ : in (a) and (b), $Y$ and $X$ are plotted versus the load $f$, respectively while in (c) the parametric curve $(Y(f), X(f))$ is shown. The dashed and double dotted linestyles denote the unstable parts of the branch for which one and two real modes are stable respectively. 


\section{Analytic solutions}

The numerical picture of the bifurcation diagram described in the previous section is completed by the analytic expression of the branches: (4) for $X=0$ and (5) for $X \neq 0$ within range of $f$ depending on the value of the aspect ratio stated in Proposition 4.2. The details of the derivation of the solutions are in appendix $\S 7$.

\subsection{The vertical snap-through}

The case $X=0$ deserves the straightforward analysis 7.2. The corresponding analytic expression of Branch \#1 is such that:

$$
\begin{aligned}
X & =0 \\
f & =\frac{2 r}{\sqrt{1+r^{2}}}(Y-1)\left(1-\frac{\sqrt{1+r^{2}}}{\sqrt{r^{2}(Y-1)^{2}+1}}\right) .
\end{aligned}
$$

\subsection{The conchoidal solutions}

The case $X \neq 0$ is detailed in 7.3. The corresponding analytic expression of Branch \#423-1 $(X<0)$ and Branch \#423-2 $(X>0)$ are such that:

$$
\begin{aligned}
& Y-1=\quad \frac{\lambda}{r}\left(1-\frac{r^{2}+1}{\left(1+\lambda^{2}\right)^{2}}\right) \quad \equiv \hat{Y}, \\
& X= \pm \frac{\sqrt{1+r^{2}}}{r \sqrt{1+\lambda^{2}}}\left(1-\frac{\lambda^{2}\left(r^{2}+1\right)}{\left(\lambda^{2}+1\right)^{3}}\right)^{1 / 2} \equiv X^{ \pm} \text {, }
\end{aligned}
$$

where

(6) $\lambda \equiv \frac{r f L}{2}=\frac{f \sqrt{1+r^{2}}}{2}$.

Following Lemma 7.3 and the solution (5), the definition range of $\lambda$ depends on the value of the aspect ratio as stated in the following proposition proved in $\S 7.4$.

The definition of range of the solution is such that

$$
\begin{aligned}
& \text { If } \left.r<r_{r s} \text { then } f \in\right]-f_{c}, f_{c}[, \\
& \text { If } \left.\left.r=r_{r s} \text { then } f \in\right]-f_{c},-f_{r s}\right] \cup\left[-f_{r s}, f_{r s}\right] \cup\left[f_{r s}, f_{c}[\text {, }\right. \\
& \text { If } \left.\left.r>r_{r s} \text { then } f \in\right]-f_{c},-f_{s}\right] \cup\left[-f_{r}, f_{r}\right] \cup\left[f_{s}, f_{c}[,\right.
\end{aligned}
$$

where

$$
f=\frac{2}{\sqrt{r^{2}+1}} \lambda=\frac{2}{\sqrt{r^{2}+1}} \sqrt{\frac{1-v}{v}}, \quad v \equiv\left(1+\lambda^{2}\right)^{-1}
$$


and with

$$
\lambda_{c}=\sqrt{-1+\sqrt{1+r^{2}}}
$$

$$
\begin{aligned}
& r_{r s}=\frac{\sqrt{23}}{2}, v_{r s}=\frac{25-2 r_{r s}^{2}}{3\left(1+r_{r s}^{2}\right)}, \\
& r>r_{r s}, v_{s}=\frac{2}{3} \cos \frac{1}{3}\left(\arccos \left(\frac{2 r^{2}-25}{2\left(r^{2}+1\right)}\right)-2 \pi\right)+\frac{1}{3}, \\
& v_{r}=\frac{2}{3} \cos \frac{1}{3} \arccos \left(\frac{2 r^{2}-25}{2\left(r^{2}+1\right)}\right)+\frac{1}{3} .
\end{aligned}
$$

\section{Connection scenario}

The present section intends to complete the global bifurcation diagram carried out with numerical tools in $\S 3$. The critical values of the parameters are explicitly derived in $\S 5.1$ from the conchoidal solution in $\S 4$. The scenario of connection is studied where a connection refers to the emergence of the separatrices of two families of curves depending on a control parameter. The connection scenario in $\S 5.3$ will result from the construction of the Ehresmann Connection [19] (See [20]) naturally associated with the problem in $§ 5.2$ and which is such that:

$$
d s^{a}=-A_{\alpha}^{a} d r^{\alpha}
$$

where $s^{a}$ and $r^{\alpha}$ denote the vertical and horizontal components of the connection respectively, namely:

$$
\left(\begin{array}{c}
s^{1} \\
s^{2}
\end{array}\right) \equiv\left(\begin{array}{c}
X \\
f
\end{array}\right), \quad\left(\begin{array}{c}
r^{1} \\
r^{2}
\end{array}\right) \equiv\left(\begin{array}{c}
t \\
\hat{Y}
\end{array}\right)
$$

\subsection{Critical values}

Following Proposition 4.2, $r_{r s}$ defined in (8-a) is the critical value of the aspect ratio of the connection of Branches \#423-1 and \#423-2 with Branch \#1. The branches respectively correspond to the analytic solutions $\left(X^{-}, Y\right),\left(X^{+}, Y\right)$ and $(X=0, Y)$ defined in (4) and (5). The so-called connection aspect ratio $r_{r s}$ is a global critical value of the parameter. Below the connection threshold, the three branches \#1, \#423-1 and \#423-2 exist and are disjoint.

The $\mathcal{C}^{1}$ connection is realized for $r=r_{r s}$ with $|\lambda| \in\left[0, \lambda_{r s}\right] \cup\left[\lambda_{r s}, \lambda_{c}[\right.$. As defined in (56-b), the polynomial $\theta$ is null at $\lambda_{r s}=\sqrt{2} / 2$, that is $X^{ \pm}=0$. Hence, Branches \#423-1 and \#423-2 both connect on Branch \#1 through two points corresponding to the critical loads such that

$$
f_{r s}^{ \pm}= \pm \frac{2 \lambda_{r s}}{\sqrt{1+r_{r s}^{2}}}= \pm \frac{2 \sqrt{6}}{9},
$$


as it is depicted in Figure 2.

Using the parametrization $(r, \lambda) \mapsto(X(r, \lambda), Y(r, \lambda))$, the expressions (56) and (57) lead to

$$
\begin{array}{r}
\partial_{\lambda} X^{ \pm}=\mp \frac{1+r^{2}}{r} \sqrt{v^{3}(1-v)} \frac{\zeta(v)}{\sqrt{v \theta(v)}} \\
\text { with } \zeta(v) \equiv 4 v^{3}-3 v^{2}+\frac{1}{1+r^{2}}=\theta(v)+v \theta^{\prime}(v) \\
\partial_{\lambda} Y=\frac{v^{3}}{r} F\left(\frac{1}{v}\right)
\end{array}
$$

where $v \in] 0,1]$ at the most. The functions $X^{ \pm}$and $\partial_{\lambda} X^{ \pm}$(resp. $Y$ and $\partial_{\lambda} Y$ ) are even and odd (resp. odd and even). Elementary computations show that $\zeta$ has positive roots if and only if $r \geq r_{k p}=\sqrt{3}$ such that :

$$
\begin{gathered}
v_{k}=\frac{1}{2} \cos \frac{\Psi-2 \pi}{3}+\frac{1}{4}<v_{c}, \\
v_{p}=\frac{1}{2} \cos \frac{\Psi}{3}+\frac{1}{4}
\end{gathered}
$$

with $\Psi=\arccos \frac{r^{2}-7}{\left(r^{2}+1\right)}$.

In a nutshell, the classification of the roots of $\theta$ and $\zeta$ is

$$
0<v_{k}<v_{c}=\frac{1}{\sqrt{1+r^{2}}}<v_{s} \leq v_{p} \leq v_{r} \leq 1
$$

As far as the behaviour of $X^{ \pm}(r, \lambda)$ at the critical value $\lambda=\lambda_{r s}$ is under focus and as long as $v_{r s}$ is a root for both $\zeta(v)$ and $\theta(v)$, the roots of these functions should be studied. Precisely:

$$
\begin{aligned}
& \theta(v)=0 \Longleftrightarrow \tau^{2}=f(v) \equiv v^{2}(1-v) \\
& \zeta(v)=0 \Longleftrightarrow \tau^{2}=g(v) \equiv v^{2}(3-4 v)
\end{aligned}
$$

where $\tau^{2} \equiv \frac{1}{1+r^{2}}$.

Figure 5 depicts the roots of $\theta$ and $\zeta$ leaning on the curves $f(v)$ and $g(v)$ respectively. For example, let us consider the primary ordinate $\tau^{2}$ on the left side of Figure 5 -a. The polynomial $\zeta$ has two roots: $v_{k}$ and $v_{p}$ and $\theta$ has no real positive roots. As $\tau^{2}=v_{c}^{2}$, the corresponding abscissa on the curve $k(v)$ is $v_{c}$ and the interval where $X^{ \pm}$is not defined is hatched. Eventually, the corresponding value of the aspect ratio is the secondary ordinate of this abscissa $v_{c}$ on the curve $h(v)$ on the right edge of the figure. The corresponding propositions and proofs are detailed in appendix 8.

One may remark that the map $(r, v) \mapsto X^{ \pm}(r, v)$ is not $\mathcal{C}^{1}$ in the vicinity of $\left(r_{r s}, v_{r s}\right)$, that is

$$
\lim _{v \rightarrow v_{r s}^{ \pm}} \partial_{\lambda} X^{ \pm}(r(v), v)=0 \neq \partial_{\lambda} X^{ \pm}\left(\lambda_{r s}, v_{r s}^{ \pm}\right)
$$




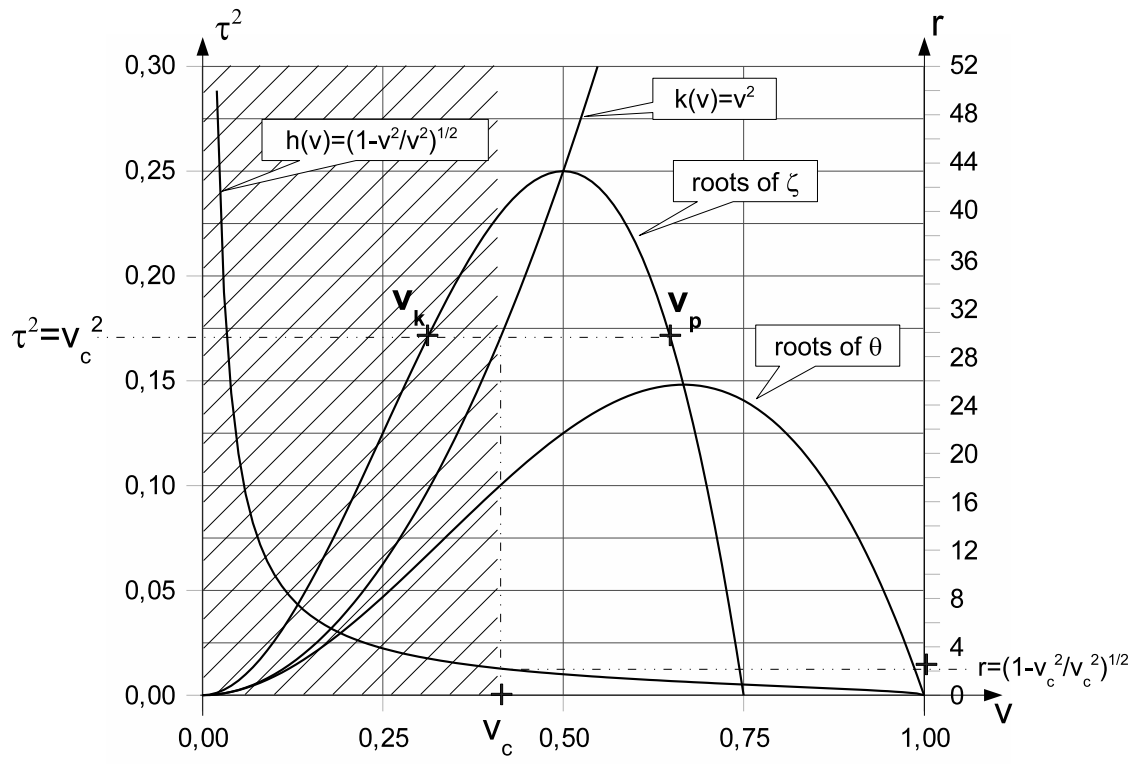

(a) $r_{k p}<r<r_{r s}$

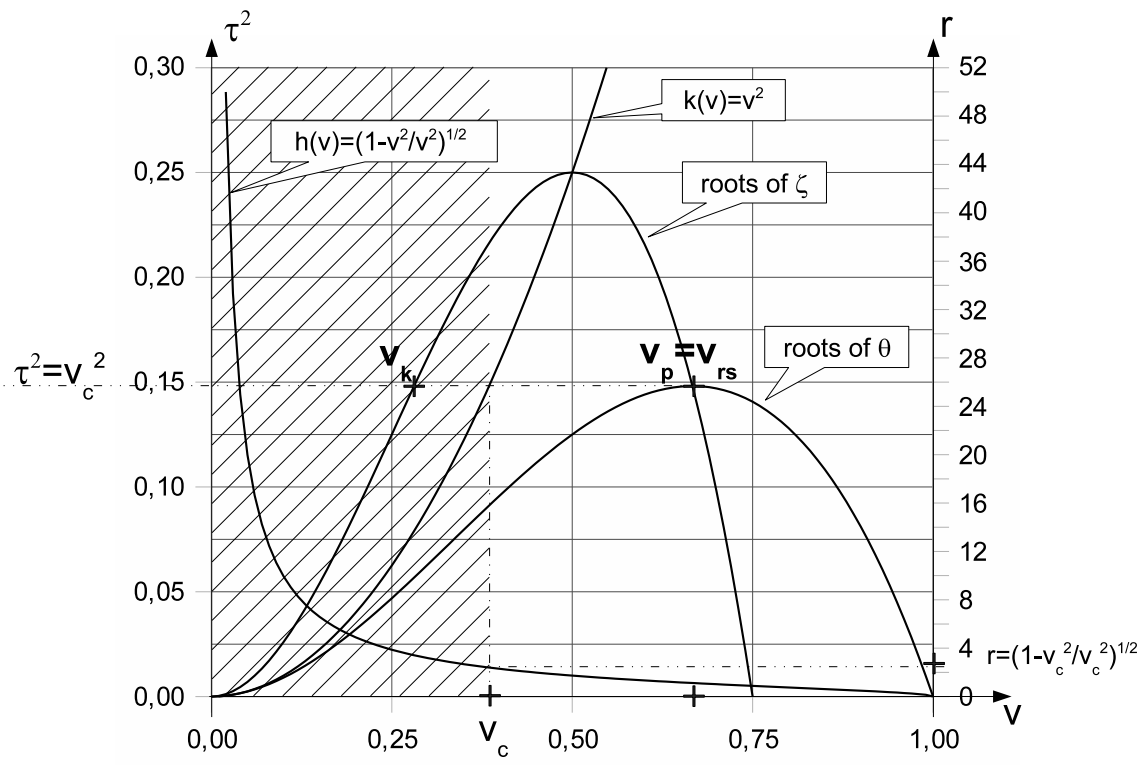

(b) $r=r_{r s}$

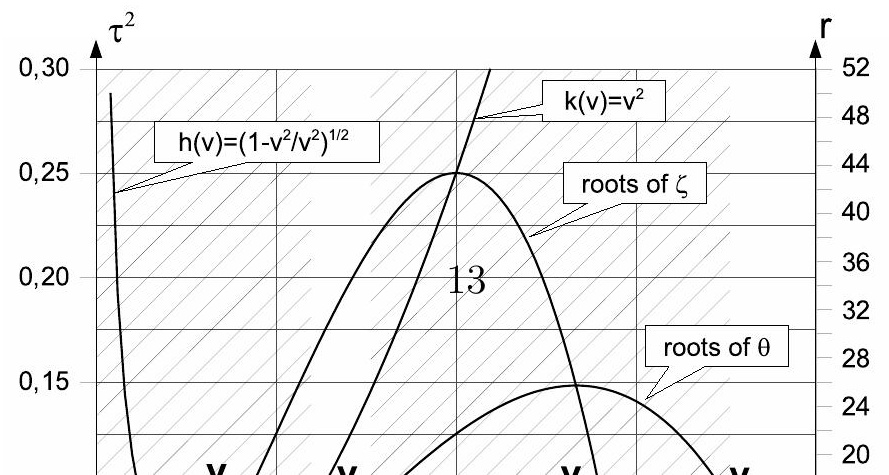


Let us consider the shallow configuration. If $\left.\left.r<r_{r s}, v \in\right] v_{c}, 1\right]$ equivalently reads $\lambda \in\left[0, \lambda_{c}\left[\right.\right.$. The curves $\left(X^{+}(\lambda), Y(\lambda)\right)$ and $\left(X^{-}(\lambda), Y(\lambda)\right)$ are $\mathcal{C}^{1}$ distinct branches. They are maximal curves. Taking into account the symmetries of (56), we focus on $X^{+}$and $\lambda>0$. There are obviously two cases: if the value of $\lambda$ is either greater or lower than $\sqrt{2} / 2$. The corresponding critical aspect ratio is such that:

$$
r_{i} \equiv \frac{\sqrt{5}}{2}
$$

The critical value $r_{i}$ is the limit between concave and corbelled $X^{ \pm}$-curves. Therefore, we may distinguish three caracteristic subsets of the aspect ratio (See Fig. 6). In the range $0<r<r_{k p}, X^{+\prime}(\lambda)>0 . X^{+}$is a concave or corbeled curve if $r$ is less or more than $r_{i}$. It has been already described in $\S 3.1$ where Figure 2 -c depicts $X>0$, i.e. $X^{+}$, as a function of the load $f$ for values of the aspect ratio within the range $r \in[0.7,2.5]$. We focus on the east quadrant: $\lambda>0$, that is $f>0$. The critical value $r_{i}$ is the limit between concave and corbelled $X$-curves. Equivalently, it is the threshold of invagination of the parametric curve (See Figure 2-d). In the range $r_{k p}<r<r_{r s}$, the polynomial $\zeta(v)$ has one real root $v_{p}$ within the range of definition of $v$. Thus, $X^{+\prime}(\lambda)$ has two roots $\left\{0, \lambda_{p}\right\}$ and $X^{+}$is still a corbeled curve as illustrated in Figure 6-c.

Based on the previous asymptotic analysis, the conchoidal solutions are in the set of piecewise $\mathcal{C}^{1}$ curves depending on the value of the aspect ratio. For all aspect ratio, the curve $(X(\lambda), Y(\lambda))$ is piecewise $\mathcal{C}^{1}$ and the set where it is $\mathcal{C}^{1}$ is kwown. Therefore, it remains to glue the $\mathcal{C}^{1}$ branches together.

Firstly, let us focus on the shallow case, that is if $r<r_{r s}$ or $\lambda \in\left[0, \lambda_{c}[\right.$. There are three $\mathcal{C}^{1}$ disjoint branches: \#423-1 \#423-2 and \#1. They respectively correspond to the analytic solutions $\left(X^{-}, Y\right),\left(X^{+}, Y\right)$ and $(X=0, Y)$ defined in (4) and (5) that we shortly denote: $X^{-}, X^{+}$and $(X=0)$ with

$$
\begin{aligned}
X^{+}\left(-\lambda_{c}<\lambda<+\lambda_{c}\right) & \equiv \# 423-2 \\
& \equiv \# 4-2 \quad \cup \quad \# 2-2 \quad \cup \quad \# 3-2, \\
X^{-}\left(-\lambda_{c}<\lambda<\lambda_{c}\right) & \equiv \# 423-1 \\
& \equiv \# 4-1 \quad \cup \quad \# 2-1 \quad \cup \quad \# 3-1 .
\end{aligned}
$$

Secondly, let us consider the connection for which $r=r_{r s}$. The tangent $\left(X^{\prime}(\lambda), Y^{\prime}(\lambda)\right)$ is continuous on the definition range $\lambda \in\left[0, \lambda_{r s}\right] \cup\left[\lambda_{r s}, \lambda_{c}\left[\right.\right.$. Thus, $X^{ \pm}(\lambda)$ are not $\mathcal{C}^{1}$ on $\left[0, \lambda_{c}\left[\right.\right.$. Rather, we have six $\mathcal{C}^{1}$ pieces such that

$$
\begin{array}{ccc}
X^{+}\left(-\lambda_{c}<\lambda<-\lambda_{r s}\right), & X^{+}\left(-\lambda_{r s}<\lambda<\lambda_{r s}\right), & X^{+}\left(\lambda_{r s}<\lambda<\lambda_{c}\right), \\
X^{-}\left(-\lambda_{c}<\lambda<-\lambda_{r s}\right), & X^{-}\left(-\lambda_{r s}<\lambda<\lambda_{r s}\right), & X^{-}\left(\lambda_{r s}<\lambda<\lambda_{c}\right) .
\end{array}
$$

Respectively, they could be equivalently denoted

$$
\# 4-2, \quad \# 2-2, \quad \# 3-2, \quad \# 4-1, \quad \# 2-1, \quad \# 3-1 .
$$



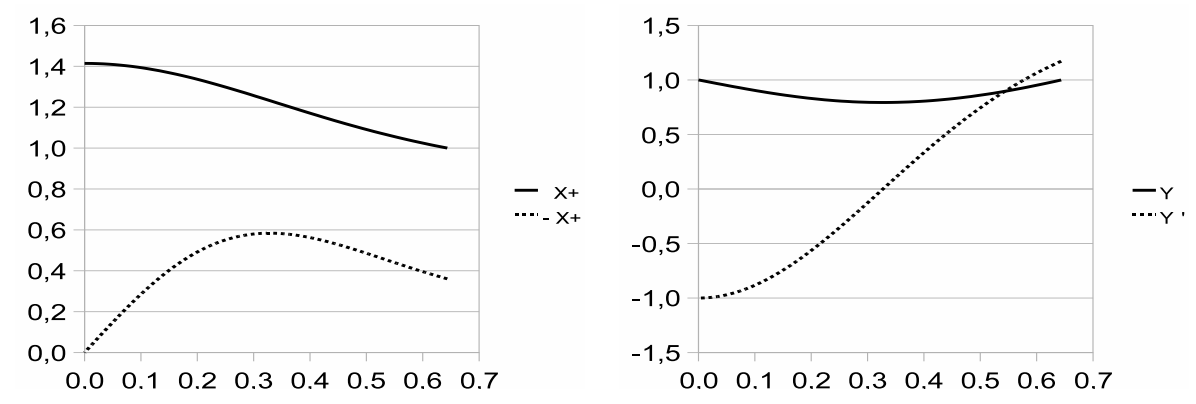

(a) $r<r_{i}$
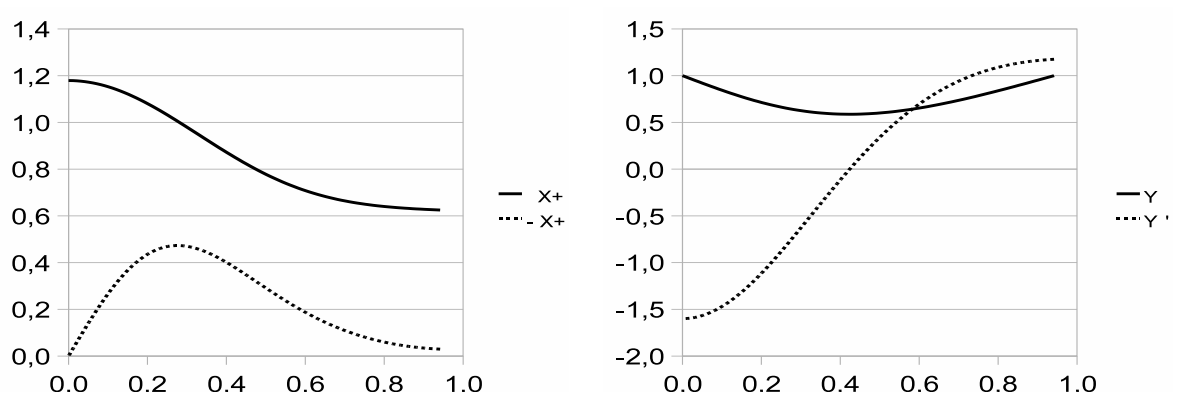

(b) $r_{i}<r<r_{k p}$
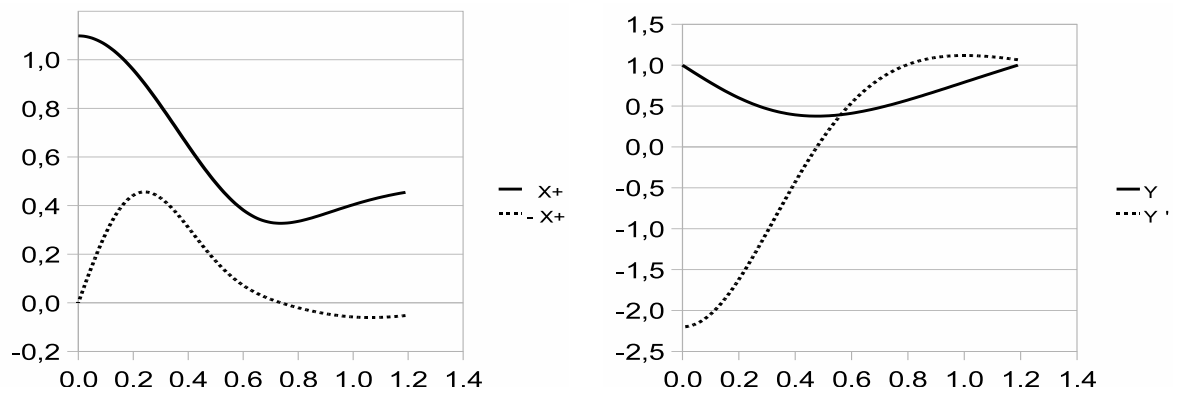

(c) $r_{k p}<r<r_{r s}$
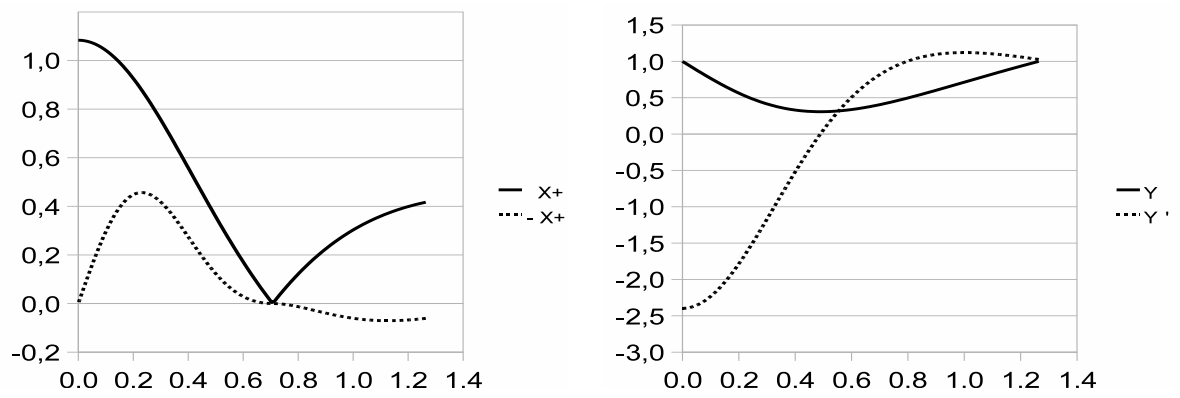

(d) $r=r_{r s}$
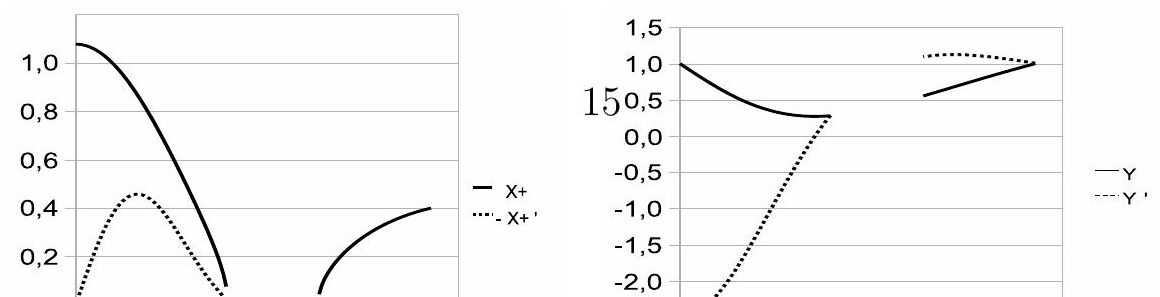
Moreover,

$$
\begin{array}{r}
\lim _{\lambda \mapsto \lambda_{r s}{ }^{-}} X^{+^{\prime}}(\lambda)=\lim _{\lambda \mapsto \lambda_{r s}{ }^{+}} X^{-^{\prime}}(\lambda) \\
X^{+}\left(\lambda_{r s}\right)=X^{-}\left(\lambda_{r s}\right) .
\end{array}
$$

The same holds for $\lambda_{r s}$ replaced by $-\lambda_{r s}$. Thus $X^{+}$and $X^{-}$are glued together at $\lambda=\lambda_{r s}$ and $\lambda=-\lambda_{\text {rs }}$ as a $\mathcal{C}^{1}$ curve on $]-\lambda_{c}, \lambda_{c}$. Exchanging the roles played by $X^{+}$and $X^{-}$, $X^{+}$and $X^{-}$are glued together at $\lambda=\lambda_{r s}$ and $\lambda=-\lambda_{r s}$ as $\mathcal{C}^{1}$ curve on $]-\lambda_{c}, \lambda_{c}$ [ which is the symmetric of the previous one with respect to $X=0$. Eventually, there are three $\mathcal{C}^{1}$ connected branches: $X=0$, and the separatrices $\mathcal{S}^{+}$and $\mathcal{S}^{-}$such that

$$
\begin{aligned}
& \mathcal{S}^{+} \equiv X^{+}\left(-\lambda_{c}<\lambda<-\lambda_{r s}\right) \cup X^{-}\left(-\lambda_{r s}<\lambda<\lambda_{r s}\right) \quad \cup \quad X^{+}\left(\lambda_{r s}<\lambda<\lambda_{c}\right) \\
& \equiv \# 4-2 \quad \cup \# 2-1 \quad \cup \# 3-2 \text {, } \\
& \mathcal{S}^{-} \equiv X^{-}\left(-\lambda_{c}<\lambda<-\lambda_{r s}\right) \cup X^{+}\left(-\lambda_{r s}<\lambda<\lambda_{r s}\right) \cup X^{-}\left(\lambda_{r s}<\lambda<\lambda_{c}\right) \\
& \equiv \# 4-1 \quad \cup \quad \# 2-2 \quad \cup \quad \# 3-1 .
\end{aligned}
$$

Thirdly, if $r>r_{r s}$ there are four $\mathcal{C}^{1}$ connected branches: $X=0$ and

$$
\begin{aligned}
\# 4 & \equiv X^{+}\left(-\lambda_{c}<\lambda<-\lambda_{s}\right) & \cup & X^{-}\left(-\lambda_{c}<\lambda<-\lambda_{s}\right) \\
& \equiv \# 4-2 & \cup & \# 4-1, \\
\# 2 & \equiv X^{+}\left(-\lambda_{r}<\lambda<\lambda_{r}\right) & \cup & X^{-}\left(-\lambda_{r}<\lambda<\lambda_{r}\right) \\
& \equiv \# 2-2 & \cup & \# 2-1, \\
\# 3 & \equiv X^{+}\left(\lambda_{s}<\lambda<\lambda_{c}\right) & \cup & X^{-}\left(\lambda_{r}<\lambda<\lambda_{c}\right) \\
& \equiv \# 3-2 & \cup & \# 3-1,
\end{aligned}
$$

This achieves the description of the connection of the disjoint branches and thus, of the bifurcation diagram.

\subsection{Connection}

The present paragraph shows that the connection previously described is actually an Ehresmann connection [19]. In this appropriate framework, the admissible scenarii will be modelized in $\S 5.3$. The conchoidal solutions of $(1,2)$ are locally a smooth function of $(t, \hat{Y})$ if

$$
\delta \equiv \hat{Y}^{2}\left(\frac{1}{\hat{L}_{1}^{3}}+\frac{1}{\hat{L}_{2}^{3}}\right)-\frac{2}{L} \neq 0
$$


where $\hat{Y}$ was defined in (5).Proof. The system $(1,2)$ equivalently reads:

$$
\begin{aligned}
\hat{R}_{1} \sqrt{(X+t)^{2}+\hat{Y}^{2}}-1 & =0, \\
\hat{R}_{2} \sqrt{(X-t)^{2}+\hat{Y}^{2}}-1 & =0, \\
\left(\hat{R}_{1}-R\right)(X+t)+\left(\hat{R}_{2}-R\right)(X-t) & =0, \\
\left(\hat{R}_{1}-R\right) \hat{Y}+\left(\hat{R}_{2}-R\right) \hat{Y}+f & =0, \\
R \sqrt{t^{2}+1}-1 & =0,
\end{aligned}
$$

with

$$
\hat{R}_{1} \equiv \frac{1}{\hat{L}_{1}}, \quad \hat{R}_{2} \equiv \frac{1}{\hat{L}_{2}}, \quad R \equiv \frac{1}{L_{1}}=\frac{1}{L_{2}} .
$$

Let us consider the vector of the unknowns $\bar{U}$ and of the parameters $\Lambda$ such that

$$
\begin{aligned}
\bar{U}^{t} & \equiv\left(\hat{R}_{1}, \hat{R}_{2}, X, f, R\right) \equiv\left(U_{1}, U_{2}, R\right), \\
\Lambda^{t} & \equiv(t, \hat{Y}),
\end{aligned}
$$

with $U \equiv\left(U_{1}, U_{2}\right) \in \mathbb{R}^{2} \times \mathbb{R}^{2}$ and $\Lambda \in \mathbb{R}^{2}$ and the general form of the system (14):

$$
B(U, R, \Lambda)=0
$$

Following the Implicit Funtion Theorem, the system (14) is a local diffeomorphism if $d B$ is non-singular. Direct computation yields the following reduced form of the block decomposition of the differentiated identity:

$$
\begin{aligned}
d B & \equiv J d U+K d \Lambda \\
& =\left(\begin{array}{ll}
J_{11} & J_{12} \\
J_{21} & J_{22}
\end{array}\right)\left(\begin{array}{l}
d U_{1} \\
d U_{2}
\end{array}\right)+\left(\begin{array}{c}
K_{1} \\
K_{2}
\end{array}\right) d \Lambda=0 .
\end{aligned}
$$

Notice that

$$
\hat{L}_{1} \hat{L}_{2}=0 \Longleftrightarrow \hat{Y}=0 \quad \text { and } \quad X^{2}=t^{2} .
$$

Substituting $\hat{Y}=0$ and $X=t$ (resp. $\hat{Y}=0$ and $X=-t$ ) in equation(14-a) (resp. (14-b)), leads to a contradiction. Thus, $J_{11}$ is invertible and the solution of (18) is such that

$$
\begin{aligned}
d U_{1} & =-J_{11}^{-1}\left(K_{1} d \Lambda+J_{12} d U_{2}\right), \\
\tilde{J} d U_{2} & =
\end{aligned}
$$

where $\delta$ the determinant of $\tilde{J}$ is such that

$$
\delta \equiv \hat{Y}^{2}\left(\hat{R}_{1}^{3}+\hat{R}_{2}^{3}\right)-2 R
$$


According to the implicit function theorem, if $\delta$ is not zero then the solution of the system is locally a smooth function of $(t, Y)$. This achieves the proof.

The conchoidal solutions of $(1,2)$ are locally smooth functions of $(t, Y)$ for $X \rightarrow 0$ if $r<r_{r s}$. The system exhibits one or two singular points if the value of the aspect ratio $r$ is equal or greater than the threshold $r_{r s}$ respectively. Proof. Let us focus on the plane $X=0$ where:

$$
\begin{aligned}
\hat{L} & \equiv \hat{L}_{1}=\hat{L}_{2}=\sqrt{\hat{Y}^{2}+t^{2}}, \\
\hat{R} & =\hat{L}^{-1}, \\
\delta & =-2\left(t^{2} \hat{R}^{3}-\hat{R}+R\right) \equiv-2 \sigma(\hat{R}) .
\end{aligned}
$$

The variations of $\sigma(\hat{R})$, immediatly imply that

$$
\sigma(\hat{R})=\sigma^{\prime}(\hat{R})=0 \Longleftrightarrow \quad r=\frac{\sqrt{23}}{2} \equiv r_{r s} \quad \text { and } \quad \hat{R}=\frac{1}{t_{r s} \sqrt{3}}=\frac{r_{r s}}{\sqrt{3}} \equiv \hat{R}_{r s} .
$$

Thus, system (14) exhibits none, one or two singular points as a function of $(t, \hat{Y})$ in the plan $X=0$ if the value of the aspect ratio $r$ is lower, equal or greater than the threshold $r_{r s}$. This achieves the proof.

The singular points as $X \rightarrow 0$ of any parametrization of the conchoidal solutions with $f^{\prime}(s) \neq 0$, are such that

$$
\frac{d X}{d f}=\frac{1}{f_{ \pm}^{\prime}\left(s_{r s}\right)} \frac{d t^{\prime}}{d X}(X=0) \quad \text { if } \quad r=r_{r s} \equiv r\left(s_{r s}\right)
$$

where the index \pm denotes the two symmetrical values of the derivative $f^{\prime}(s)$. Proof. For $t=t_{r s}$ and $\hat{R}=\hat{R}_{r s}$, we focus on the vicinity of the connection point $(X=0)$ for which

$$
\begin{aligned}
\bar{U}^{t} & \equiv\left(\hat{R}_{r s}, \hat{R}_{r s}, X \rightarrow 0, f_{r s}, R_{r s}\right), \\
\Lambda^{t} & \equiv\left(t_{r s}, \hat{Y}_{r s}\right) .
\end{aligned}
$$

with

$$
R_{r s} \equiv \frac{1}{\sqrt{t_{r s}^{2}+1}}, \quad f_{r s} \equiv 2 \sqrt{2} t_{r s}\left(\frac{1}{\sqrt{1+t_{r s}^{2}}}-\frac{1}{t_{r s} \sqrt{3}}\right), \quad \hat{Y}_{r s} \equiv \sqrt{2} t_{r s} .
$$

Following corollary 5.2, the singularity is a point. Thus, the vicinity, without singularity other than that considered, does exist. Consider a parametrization of a branch of (14): $s \mapsto(U(s), \Lambda(s))$. Differentiating (14) with respect to $s$ yields:

$$
J(U(s), \Lambda(s)) U^{\prime}(s)+K(U(s), \Lambda(s)) \Lambda^{\prime}(s)=0
$$

and, specifically:

$$
\tilde{J}(U(s), \Lambda(s))\left(\begin{array}{c}
X^{\prime}(s) \\
f^{\prime}(s)
\end{array}\right)+\tilde{K}(U(s), \Lambda(s))\left(\begin{array}{c}
t^{\prime}(s) \\
\hat{Y}^{\prime}(s)
\end{array}\right)=0
$$


Given any function, vector or matrix $g(X)$, let $g(X)=g_{e}(X)+g_{o}(X)$ denotes the decomposition of $g$ into even and odd parts respectively. The differential equality (22) leads to

$$
\begin{aligned}
& \tilde{J}_{e}\left(\begin{array}{c}
X_{e}^{\prime} \\
f_{e}^{\prime}
\end{array}\right)+\tilde{J}_{o}\left(\begin{array}{c}
X_{o}^{\prime} \\
f_{o}^{\prime}
\end{array}\right)+\tilde{K}_{e}\left(\begin{array}{c}
t_{e}^{\prime} \\
Y_{e}^{\prime}
\end{array}\right)+\tilde{K}_{o}\left(\begin{array}{c}
t_{o}^{\prime} \\
Y_{o}^{\prime}
\end{array}\right)=0 \\
& \tilde{J}_{e}\left(\begin{array}{c}
X_{o}^{\prime} \\
f_{o}^{\prime}
\end{array}\right)+\tilde{J}_{o}\left(\begin{array}{c}
X_{e}^{\prime} \\
f_{e}^{\prime}
\end{array}\right)+\tilde{K}_{e}\left(\begin{array}{c}
t_{o}^{\prime} \\
Y_{o}^{\prime}
\end{array}\right)+\tilde{K}_{o}\left(\begin{array}{c}
t_{e}^{\prime} \\
Y_{e}^{\prime}
\end{array}\right)=0 .
\end{aligned}
$$

As $\delta$ is even with respect to $X$, in the vicinity of the critical value $s=s_{r s}$, we have

$$
\tilde{J}_{e} \sim\left(\begin{array}{cc}
X^{2} \sigma_{1}(X) & 0 \\
0 & 1
\end{array}\right)
$$

for some function $\sigma_{1}$ that does not vanish. Therefore, the equation (23-a) equivalently reads for $s=s_{c}$ :

$$
\begin{aligned}
\sigma_{1}(X) X_{e}^{\prime} & \left.\sim 2 t_{r s}\left(3 \hat{Y}_{r s}^{2} \hat{R}_{r s}^{5}-\hat{R}_{r s}^{3}\right) \frac{d t_{o}^{\prime}}{d X}\right|_{X=0}+\left.2 \hat{R}_{r s}\left(\hat{R}_{r s}^{2}-3 t_{r s}^{2} \hat{R}_{r s}^{4}\right) \frac{d \hat{Y}_{o}^{\prime}}{d X}\right|_{X=0} \\
& =\left.\frac{2}{3 \sqrt{3} t_{r s}^{2}} \frac{d t_{o}^{\prime}}{d X}\right|_{X=0} \\
f_{e}^{\prime} & \sim 2 t_{r s} \hat{Y}_{r s}\left(\hat{R}_{r s}^{3}-R_{r s}^{3}\right) t_{e}^{\prime}+2\left(R_{r s}-t_{r s}^{2} \hat{R}_{r s}^{3}\right) \hat{Y}_{e}^{\prime} \\
& = \pm 2 \sqrt{2} t_{r s}^{2}\left(\hat{R}_{r s}^{3}-R_{r s}^{3}\right) t_{e}^{\prime}+2\left(R_{r s}-t_{r s}^{2} \hat{R}_{r s}^{3}\right) \hat{Y}_{e}^{\prime} \equiv f_{e}^{\prime \pm}
\end{aligned}
$$

The same holds for the equation (23-b) substituting the index $o$ by $e$ and $e$ by $o$. Therefore, there results

$$
\frac{X^{\prime}\left(s_{r s}\right)}{f^{\prime}\left(s_{r s}\right)}=\frac{d X}{d f}\left(f_{r s}\right) \sim \frac{X_{e}^{\prime}}{f^{\prime \pm}}
$$

Notice that if $X \rightarrow 0$ and $t \neq t_{r s}$, then, $\delta \neq 0$ implies

$$
\begin{aligned}
& \delta X_{e}^{\prime} \sim-X^{2}\left(\left.\hat{Y}^{2} \frac{d}{d X}\left(\hat{R}_{1}^{3}-\hat{R}_{2}^{3}\right)\right|_{X=0}+2 t R^{3}\right) \frac{d t_{o}^{\prime}}{d X}(0)+ \\
& +X^{2}\left(2 \hat{R}_{1}^{3}+\left.t \frac{d}{d X}\left(\hat{R}_{1}^{3}-\hat{R}_{2}^{3}\right)\right|_{X=0}\right) \frac{d \hat{Y}_{o}^{\prime}}{d X}(0)=\mathcal{O}\left(X^{2}\right)
\end{aligned}
$$

and thus:

$$
\frac{X^{\prime}}{f^{\prime}}=\frac{d X}{d f} \sim \frac{X_{e}^{\prime}}{f_{e}^{\prime}}=\frac{\mathcal{O}\left(X^{2}\right)}{f_{e}^{\prime}} \rightarrow 0
$$

as $\left.\lim _{X \rightarrow 0} f_{e}^{\prime}\right|_{s=s_{r s}} \neq 0$ because the both coefficients with respect to $t_{e}^{\prime}$ and $\hat{Y}_{e}^{\prime}$ are not null.

Set the following vectors after the coefficients of $(24)$ :

$$
\mathcal{K}_{11}=\left(\frac{2}{3 \sqrt{3} t_{r s}^{2} \sigma_{1}(0)}\right) \quad \mathcal{K}_{22}=\left(\begin{array}{c} 
\pm 2 \sqrt{2} t_{r s}^{2}\left(\hat{R}_{r s}^{3}-R_{r s}^{3}\right) \\
2\left(R_{r s}-t_{r s}^{2} \hat{R}_{r s}^{3}\right)
\end{array}\right)
$$


and consider any connection defined by the differential equality:

$$
d \Lambda=-\left(\begin{array}{cc}
b_{1}^{T}(X) & c_{1}^{T}(X) \\
b_{2}^{T}(X) & c_{2}^{T}(X)
\end{array}\right)\left(\begin{array}{c}
d U_{2} \\
d \Lambda
\end{array}\right)
$$

where $b_{a} \in \mathbb{R}^{2}$ and $c_{a} \in \mathbb{R}^{2}$ are vector fields on $\mathbb{R}^{2}$. The following corollary states the Ehresmann connection naturally associated with the problem.

Consider the connection (27) and assume that

$$
b_{a}(X)=b_{a}(0)+\mathcal{O}\left(X^{2}\right), \quad a \in\{1,2\} .
$$

Then, the connection $(19-\mathrm{b})$ is $(d X, d f)^{T}$ such that:

$$
\begin{array}{r}
-\tilde{J}^{-1} \tilde{K}\left(\begin{array}{c}
d t \\
d \hat{Y}
\end{array}\right) \quad \text { if } \quad X \neq 0 \\
\left(\begin{array}{cc}
\mathcal{K}_{11}^{T} & 0 \\
0 & \mathcal{K}_{22}^{T}
\end{array}\right)\left(\begin{array}{c}
c^{\prime}(0) \\
I_{2}
\end{array}\right)\left(\begin{array}{c}
d t \\
d \hat{Y}
\end{array}\right) \quad \text { as } \quad X \rightarrow 0
\end{array}
$$

where we have set

$$
c(X)=\left(\begin{array}{c}
c_{1}^{T}(X) \\
c_{2}^{T}(X)
\end{array}\right) \in \mathbb{R}^{2 \times 2}
$$

with $c^{\prime}(0)$ the derivative of the matrix $c$ with respect to $X$ and $I_{2} \in \mathbb{R}^{2 \times 2}$ denotes the identity matrix. Proof. With the notations (16), the identity (27) equivalently reads:

$$
d \Lambda=-\eta\left(X ; d U_{2}, d \Lambda\right)
$$

where the right hand side of (30) is a differential form with coefficients depending on $X$. Then, (24) equivalently reads:

$$
d U_{2} \sim\left(\begin{array}{cc}
\mathcal{K}_{11}^{T} & 0 \\
0 & \mathcal{K}_{22}^{T}
\end{array}\right)\left(\begin{array}{c}
-\frac{\partial \eta}{\partial X}\left(0 ; d U_{2}, d \Lambda\right) \\
d \Lambda
\end{array}\right) \quad \text { as } \quad X \rightarrow 0
$$

Taking into account the hypothesis (28), direct computation yields:

$$
\left(\begin{array}{c}
-\frac{\partial \eta}{\partial X}\left(X ; d U_{2}, d \Lambda\right) \\
d \Lambda
\end{array}\right)=\left(\begin{array}{c}
\mathcal{O}(X) \\
0
\end{array}\right) d U_{2}+\left(\begin{array}{c}
-c^{\prime}(0)+\mathcal{O}(X) \\
I_{2}
\end{array}\right) d \Lambda .
$$

We conclude after substitution into (31).

The existence of the connection (29) coïncides with the occurence of two values of the derivative $X^{\prime} / f^{\prime}$ as $X \rightarrow 0$, that is,

$$
\lim _{X \rightarrow 0^{+}} \frac{X^{\prime}}{f^{\prime}}=\frac{X_{e}^{\prime}}{f_{e}^{\prime+}} \neq \lim _{X \rightarrow 0^{-}} \frac{X^{\prime}}{f^{\prime}}=\frac{X_{e}^{\prime}}{f_{e}^{\prime-}} .
$$

This can be interpreted as the emergence of separatrices at $X=0$ between the two families of curves: Branches \#423-1 and \#423-2 before connection and Branches \#2, \#3 and \#4 after connection. 


\subsection{Scenario}

Taking into account of (25), the connection scenarii can be described as it follows.

If $\left|X^{\prime} / f^{\prime}\right|$ has a finite positive limit as $X \rightarrow 0$ then, the connection links two partner curves of the family of Branches \#423-1 and \#423-2. Indeed, the equation (29) yields:

$$
\lim _{X \rightarrow 0} \frac{X^{\prime}}{f^{\prime}}=\frac{\mathcal{K}_{11 a} c_{a}^{\prime}(0) \cdot \Lambda^{\prime}}{\mathcal{K}_{22} \cdot \Lambda^{\prime}}= \pm \frac{X_{e}^{\prime}}{f_{e}^{\prime \pm}}
$$

which means that the slope $X^{\prime} / f^{\prime}$ is finitely nonzero at the connection on the branch \#1 if $c^{\prime}(0) \neq 0$. Therefore, the initial curve is transformed through the connection into its partner curve.

If the limit of $\left|X^{\prime} / f^{\prime}\right|$ is zero as $X \rightarrow 0$ then the connection links Branch \#423-1 or \#423-2 either with Branch \#1 or with a partner curve in the family \#2, \#3 and \#4. As a matter of fact, the same arguments as in the proof of (29) show that, if $c(X)=\mathcal{O}\left(X^{2}\right)$, then,

$$
\frac{X^{\prime}}{f^{\prime}} \sim X^{m} \frac{\mathcal{K}_{11 a} c_{a}^{(m+1)}(0) \cdot \Lambda^{\prime}}{m ! \mathcal{K}_{22} \cdot \Lambda^{\prime}} \rightarrow 0 \quad \text { as } \quad X \rightarrow 0 .
$$

Hence, the initial curve is linked to either Branch \#1 or a partner branch in the family $\# 2$, \#3 and \#4. Initial values at $X=0$ are necessary to discriminate between both possibilities.

\section{Conclusion}

All or part of bifurcations, singularities and control theories on one hand, and of homogeneization and catastrophe theories on the other hand, enable to highlight the fundamental characteristics of nonlinear dynamics. For a system under focus, one of the first fundamental step is the determination of the most complete picture possible of the connected and disjoint subsets of the stable and unstable equilibria. In this work, the prototype of the truss arch system is considered and studied. The coexistence of disjoint subsets of equilibria is shown. The corresponding analytical solutions are derived. Moreover, it is shown that the emergence of the subcritical bifurcations of the non-shallow configuration is the result of a transcritical connection of these disjoint branches in the sense of Ehresmann [19].

This preliminary work suggests that the present approach might be used in future works on real systems for which the coexistence of disjoint subsets of equilibria have been identified or suspected. For example, three well-known cases could be considered. Firstly, one would may use the present approach for the study of identified isolated branches of steady states as in the parallel flow (e.g. [22, 23, 24, 25, 26, 27, 28, 29]). Secondly, the methodology of the present work is likely to be a good candidate for the treatment of imperfect bifurcation problems, as for example, those of the cylindrical shells or the reticulated truss shells (e.g. [1, 2, 17, 30]). Eventually, well-known examples of suspected isolated branches of steady states of industrial interest might be considered as for example, thermocapillar or rotating flows (e.g. [31, 32, 33]). 


\section{References}

[1] Nguyen, Q. S., Stability and Nonlinear Solid Mechanics John Wiley, 2000.

[2] Antman, S. S., Nonlinear Problems of Elasticity, Springer, 2005.

[3] Matkowsky, B.J., Reiss, E.L., 1977, Singular Perturbations of Bifurcations, SIAM J. Appl. Math., 33, 230-255.

[4] Dijkstra, H. A., Molemaker, M. J., 1999, Imperfections of the North-Atlantic winddriven ocean circulation: continental geometry and assymetric wind-stress, J. Mar. Res., $\mathbf{5 7}, 1-28$.

[5] Simonnet, E., Ghil, M., Ide, K., Temam, R., Wang, S., 2003, Low-frequency variability in the Shallow-Water models of the wind-driven Ocean circulation, J. Phys. Oceanogr., 33, 712-751.

[6] Golubitsky,M., Shaeffer, D., 1979, A theory for Imperfect Bifurcation via Singularity Theory, Communications on Pure and Applied Mathematics, 32, 21-98.

[7] Thom, R., Structural Stability and Morphogenesis, Addison Wesley, 1989.

[8] Cantin, Y. G., Cousin-Rittemard, N. M. M., Gruais, I., 2008, On the catastrophic bifurcation diagram of the truss arch system, C.R. Mecanique, 336, 699-703.

[9] Cousin-Rittemard, N. M. M., Gruais, I., 2007, Continuation Methods and disjoint equilibria, Revue Roumaine de Mathématiques Pures et Appliquées 52, 9-34.

[10] Gruais, I., Cousin-Rittemard, N.M.M, Dijkstra, H.A., 2005, A priori estimation of a global Homotopy Residue Continuation Method, Numerical Functional Analysis and Optimization 26, 507-521.

[11] Barenblatt, G.I., Scaling, Cambridge University Press, 2003.

[12] Allgower, E.L., Georg, K., Numerical path following, Handbook of Numerical Analysis 5 (P.G. Ciarlet and J.L. Lions Eds.), 3-208, 1997.

[13] Doedel, E., Tuckerman, L.S., Numerical Methods for Bifurcation Problems and LargeScale Dynamical Systems, IMA Volumes in Mathematics and Its Applications, 119, Springer, 2000.

[14] Kuznetsov, Iu. A., Elements of Applied Bifurcation Theory, Springer Verlag, 2004.

[15] Francis, J. G. F., 1961, The $Q R$ transformation: a unitary analogue to the $L R$ transformation. I., Comput. J. 4, 265-271.

[16] Golub, G. H. and Van Loan, C. F., Matrix Computations, Johns Hopkins Studies in Mathematical Sciences, 694 pp., 1996. 
[17] Ikeda, K., Murota, K., Imperfect bifurcation in Structures and Materials, Applied Mathematical Sciences, 149, Springer-Verlag, New-York, 2002.

[18] Thompson, J.M.T., Instabilities and Catastrophes in Science and Engineering John Wiley, 242 pp., 1982.

[19] Ehresmann, C., Les connexions infinitésimales dans un espace fibré différentiable, Colloque de topologie, 28, 29-55, Bruxelles, 1950.

Oeuvres complètes et commentées, parties I-1 et I-2, 179-205, 601 p., Ed. Andrée Ehresmann, 1984.

[20] Bloch,A.M., Krishnaprasad, P.S., Marsden, J.E., Murray, R.M., 1996, Nonholonomic Mechanical Systems with Symmetry, Arch. Rat. Mech. Anal., 136, 21-99.

[21] Attouch, H., Variational convergence for functions and operators, Applicable Mathematics Series, Pitman, 1984.

[22] Zahn, J.P., Toomre, J., Speigel,E.A., and Gough, D.O., Nonlinear cellular motion in Poiseuille channel flow, Journal of Fluid Mechanics 64, 319-345, 1974.

[23] Herbert, T., Stability of plane Poiseuille flow - theory and experiments, Fluid Dynamics and Transactions 11, 77-126, 1981.

[24] Milinazzo, F.A., Saffman, P.G., Finite-amplitude steady waves in plane viscous shear flows, Journal of Fluid Mechanics 160, 280-295, 1985.

[25] Soibelman, I., Meiron, D.I., Finite-amplitude bifurcations in plane Poiseuille flow: two-dimensional bifurcation, Journal of Fluid Mechanics, 229, 389-416, 1991.

[26] Mehta, P.G., Nonbifurcating solutions for parallel flows, Ph.D. Thesis, Cornell University, 2004.

[27] Nagata, M., Three-dimensional traveling-wave solutions in plane Couette flow, Phys. Rev. E 55, 2023 2025, 1997.

[28] Cherhabili, A., Ehrenstein, U., Finite-amplitude equilibrium states in plane Couette flow, Journal of Fluid Mechanics 342, 159-177, 1997.

[29] Barkley, D., Tuckerman, L.S., Mean flow of turbulent-laminar patterns in plane Couette flow, J. Fluid Mech. 576, 109-137, 2007.

[30] Lagnese, J.E., Schmidt, E.J., Modeling, Analysis and Control of Dynamic Elastic Multi-Link Structures, Birkhäuser, 1994.

[31] E. Chénier, C. Delcarte, G. Kasperski and G. Labrosse, Sensitivity of the liquid bridge hydrodynamics to local capillary contributions, Phys. Fluids, 14, 3109-3117, 2002.

[32] N. Cousin-Rittemard, O. Daube, P. Le Quéré, On the nature of the first bifurcation in disk systems, C.R.Acad. Sci. Paris, 326, série II b, p 359-366, 1998. 
[33] Bernsen, E., Diskstra, H.A., Wubs, F.W., Bifurcation analysis of wind-driven flows with MOM4, submitted to Ocean Modelling, 2008.

\section{Details on the analytic solution}

\subsection{Preliminary definitions}

For $Y \neq 1$, the system under focus can be seen as the equivalent linear system:

$$
\begin{aligned}
\left(X+\frac{1}{r}\right)\left(\frac{1}{\hat{L}_{1}}-\frac{1}{L}\right)+\left(X-\frac{1}{r}\right)\left(\frac{1}{\hat{L}_{2}}-\frac{1}{L}\right) & =0 \\
(Y-1)\left(\frac{1}{\hat{L}_{1}}-\frac{1}{L}\right)+(Y-1)\left(\frac{1}{\hat{L}_{2}}-\frac{1}{L}\right) & =-f,
\end{aligned}
$$

where $L$ is such that

$$
L=L_{1}=L_{2}=\frac{\sqrt{r^{2}+1}}{r}
$$

and considered together with the geometrical constraints:

$$
\hat{L}_{1}=\sqrt{\left(X+\frac{1}{r}\right)^{2}+(Y-1)^{2}}, \quad \hat{L}_{2}=\sqrt{\left(X-\frac{1}{r}\right)^{2}+(Y-1)^{2}} .
$$

Direct computation of the linear system (32) yields:

$$
\hat{L}_{1}=\frac{L}{1+\lambda a}, \quad \hat{L}_{2}=\frac{L}{1-\lambda b}
$$

where we set

$$
a \equiv \frac{\left(X-\frac{1}{r}\right)}{Y-1}, \quad b \equiv \frac{\left(X+\frac{1}{r}\right)}{Y-1}, \quad \lambda \equiv \frac{r f L}{2}=\frac{f \sqrt{1+r^{2}}}{2} .
$$

There results equivalently:

$$
\hat{L}_{1}=\frac{L(Y-1)}{Y-1-\frac{\lambda}{r}+\lambda X}, \quad \hat{L}_{2}=\frac{L(Y-1)}{Y-1-\frac{\lambda}{r}-\lambda X} .
$$

From the geometrical constraints (34) and from the solution (35) of the linear system, $\lambda$ must satisfy the following second order equation:

$$
\left(a^{2}-b^{2}\right) \lambda^{2}+2(a+b)(1+a b) \lambda+b^{2}-a^{2}=0 .
$$




\subsection{The vertical snap-through}

The case $X=0$ deserves a straightforeward analysis. From the solution (37) of the linear system on one hand and of the contitutive constraints (34) on the other hand, we get:

$$
\frac{\lambda}{r}=(Y-1)\left(1-\frac{\sqrt{1+r^{2}}}{\sqrt{r^{2}(Y-1)^{2}+1}}\right) .
$$

This is equivalent to the analytical expression of Branch \#1.

\subsection{The conchoidal solutions}

Firstly, the following lemma is shown. For $X \neq 0$, the solutions belong to the two horn surface defined by the following expression :

$$
(Y-1-d)^{2}+X^{2}=\rho^{2}
$$

where $\rho=\frac{f}{|f|} \frac{1}{2 r}\left(\lambda+\frac{1}{\lambda}\right)$ and $d=\frac{1}{2 r}\left(\lambda-\frac{1}{\lambda}\right)$. Proof. Taking the trivial condition $a-b \neq 0$ into account and, assuming that $a+b \neq 0$, that is, $X \neq 0$, the second order equation (38) also reads:

$$
\lambda^{2}+2 \lambda\left(\frac{1+a b}{a-b}\right)-1=0
$$

where ith roots are such that:

$$
\lambda^{ \pm}=-\frac{1+a b}{a-b} \pm \sqrt{1+\left(\frac{1+a b}{a-b}\right)^{2}} .
$$

According to the definitions (36) on one hand and, the second order equation (40) on the other hand, we have:

$$
\frac{1+a b}{a-b}=-\frac{r}{2}\left(Y-1+\frac{X^{2}-\frac{1}{r^{2}}}{Y-1}\right)=\frac{1-\lambda^{2}}{2 \lambda}
$$

which yields:

$$
(Y-1)^{2}+\frac{1-\lambda^{2}}{r \lambda}(Y-1)+X^{2}=\frac{1}{r^{2}}
$$

or, equivalently, the proof of lemma 7.3 is achieved.

Secondly, it is shown that for $X \neq 0$, the solutions are defined in the following proposition. For $X \neq 0$, the solutions are such that:

$$
\begin{aligned}
Y-1 & =\frac{\lambda}{r}\left(1-\frac{r^{2}+1}{\left(1+\lambda^{2}\right)^{2}}\right), \\
X & = \pm \frac{\sqrt{1+r^{2}}}{r \sqrt{1+\lambda^{2}}}\left(1-\frac{\lambda^{2}\left(r^{2}+1\right)}{\left(\lambda^{2}+1\right)^{3}}\right)^{1 / 2} \equiv X^{ \pm}
\end{aligned}
$$


where $\lambda \equiv \frac{r f L}{2}=\frac{f \sqrt{1+r^{2}}}{2}$. Proof. According to Lemma 7.3, a suitable parametrization the unknown vector $(X, Y)$ is such that

$$
X=\rho \cos \theta, \quad Y-1=\rho \sin \theta+d,
$$

where $\rho$ is a parameter and $\theta$ is thus the unique unknown.

On one hand and, noticing that the following relation holds:

$$
\rho^{2}=\frac{1}{r^{2}}+d^{2}
$$

the geometrical constraints (34) induce:

$$
\begin{aligned}
\hat{L}_{1}^{2}-\hat{L}_{2}^{2} & =4 \rho \frac{\cos \theta}{r} \\
\hat{L}_{1}^{2}+\hat{L}_{2}^{2} & =4 \rho^{2}\left(1-\frac{f}{|f|}\left(\frac{1-\lambda^{2}}{1+\lambda^{2}}\right) \sin \theta\right)
\end{aligned}
$$

where the following identity holds:

(43) $\frac{d}{\rho}=\frac{f}{|f|}\left(\frac{\lambda^{2}-1}{\lambda^{2}+1}\right)$.

On the other hand, from the direct solution (37) of the linear system we get:

$$
\begin{gathered}
\hat{L}_{1}^{2}-\hat{L}_{2}^{2}=-4 L^{2}(\rho \sin \theta+d)^{2} \frac{\left(\rho \sin \theta+d-\frac{\lambda}{r}\right) \lambda \rho \cos \theta}{\left(\left(\rho \sin \theta+d-\frac{\lambda}{r}\right)^{2}-(\lambda \rho \cos \theta)^{2}\right)^{2}} \\
\hat{L}_{1}^{2}+\hat{L}_{2}^{2}=2 L^{2}(\rho \sin \theta+d)^{2} \frac{\left(\rho \sin \theta+d-\frac{\lambda}{r}\right)^{2}+(\lambda \rho \cos \theta)^{2}}{\left(\left(\rho \sin \theta+d-\frac{\lambda}{r}\right)^{2}-(\lambda \rho \cos \theta)^{2}\right)^{2}}
\end{gathered}
$$

Taking the identities $(33,43)$ into account, we find that (42-a) and (44-a) induce:

$$
\rho\left(\frac{f}{|f|}-\sin \theta\right)=\frac{\lambda}{\left(1+\lambda^{2}\right)^{2}} \frac{r^{2}+1}{r}
$$

because $\cos \theta \neq 0$, that is, $X \neq 0$. Noticing that

$$
r \rho=\frac{f}{|f|} \frac{\left(1+\lambda^{2}\right)}{2 \lambda}
$$

thus (45) equivalently reads:

$$
\frac{f}{|f|}-\sin \theta=\frac{f}{|f|} \frac{8 \lambda^{4}}{f^{2}\left(1+\lambda^{2}\right)^{3}}
$$

Furthermore, (42-b) and (44-b) imply:

$$
1+\frac{\lambda^{2}(\cos \theta)^{2}}{\left(\sin \theta-\frac{f}{|f|}\right)^{2}}=2\left(1+\lambda^{2}\right)\left(\frac{\rho}{L}\right)^{2}\left(2 \lambda^{2}+\frac{f}{|f|}\left(1-\lambda^{2}\right)\left(\sin \theta-\frac{f}{|f|}\right)\right) \text {. }
$$


From the relation:

$$
\frac{\rho^{2}}{L^{2}}=\frac{\left(1+\lambda^{2}\right)^{2}}{4\left(r^{2}+1\right) \lambda^{2}}
$$

we immediately infere that the left hand side also reads:

$$
1+\frac{\left(\frac{f}{|f|}+\sin \theta\right)}{\left(\frac{f}{|f|}-\sin \theta\right)} .
$$

Hence, we conclude that $\frac{f}{|f|}-\sin \theta \equiv Z$ solves the second order polynomial:

$$
\frac{1}{4} \frac{\left(1+\lambda^{2}\right)^{3}\left(1-\lambda^{2}\right)}{\left(1+r^{2}\right) \lambda^{2}} Z^{2}+\frac{f}{2|f|}\left(\frac{\left(1+\lambda^{2}\right)^{3}}{\left(1+r^{2}\right)}+\lambda^{2}-1\right) Z-\lambda^{2}=0
$$

Considering both cases $f>0$ and $f<0$ separately, the roots of (47) are compatible with (46) if and only if:

$$
Z_{ \pm}=Z_{\frac{f}{|f|}}
$$

Notice that

$$
Y-1=\rho \sin \theta+d=-\rho\left(\frac{f}{|f|}-\sin \theta\right)+\rho \frac{f}{|f|}\left(\frac{2 \lambda^{2}}{1+\lambda^{2}}\right) .
$$

After substitution of (45) and according to the definition of $\rho$, we get

(48) $Y-1=\frac{\lambda}{r}\left(1-\frac{r^{2}+1}{\left(1+\lambda^{2}\right)^{2}}\right)$,

that is (5-a).

Eventually, let us consider the straightforward computation of $X^{2}$ :

$$
X^{2}=\rho\left(\frac{f}{|f|}-\sin \theta\right)\left(2 \rho \frac{f}{|f|}-\rho\left(\frac{f}{|f|}-\sin \theta\right)\right)
$$

that is $(5-b)$.

Thirdly, preliminary Lemmas 7.3 and 7.3 enable Proposition 4.2 of the definition range for $\lambda$ as it follows. The solution of the linear system satisfies the following constraints:

$$
(Y-1)\left(Y-1-\frac{\lambda}{r}+\lambda X\right)>0 \quad \text { and } \quad(Y-1)\left(Y-1-\frac{\lambda}{r}-\lambda X\right)>0 .
$$

Proof. This is an obvious consequence of the preliminary result:

(50) $\hat{L}_{1}>0$ and $\hat{L}_{2}>0$,

and of the geometrical constraints (37). Indeed, assume that $\hat{L}_{1}=0$ without loss of generality. Thus, (37) yields that

(51) $\quad Y-1=0$ 
as it is easily shown that the formulas for the linear system extend to $Y-1=0$. Hence, according to the definition (34-a) of $\hat{L}_{1}$, we infer

$$
X=-\frac{1}{r}
$$

Substituting the equality (51) in the expression (37) of $\hat{L}_{2}$, we find that $\hat{L}_{2}=0$. Then, the definition (34-b) of $\hat{L}_{2}$ induces: $X=\frac{1}{r}$, which contradicts (52), and (50) holds true. An equivalent conclusion comes if one considers $\hat{L}_{2}=0$.

The solution of the linear system (32) is defined for $\lambda$ within the range such that

$$
\lambda \in]-\lambda_{c}, 0[\cup] 0, \lambda_{c}[
$$

where $\lambda_{c}=\sqrt{-1+\sqrt{1+r^{2}}}$. It extends to $\left.\lambda \in\right]-\lambda_{c}, \lambda_{c}[$ as a solution of (32) for the most. Proof. First, notice that the analytic expression of $Y$ (48) induces:

$$
Y-1=0 \Longleftrightarrow \lambda=0 \quad \text { or } \quad \lambda= \pm \lambda_{c}
$$

where

$$
\lambda_{c}=\sqrt{-1+\sqrt{1+r^{2}}} .
$$

Hence, the solution of the linear system is defined for $\lambda \in I_{1} \cup I_{2} \cup I_{3} \cup I_{4}$ where we set

$$
\begin{gathered}
\left.I_{1}=\right]-\infty,-\lambda_{c}\left[, \quad I_{2}=\right]-\lambda_{c}, 0[, \\
\left.\left.\left.I_{3}=\right] 0,+\lambda_{c}\right], \quad I_{4}=\right] \lambda_{c},+\infty[.
\end{gathered}
$$

Furthermore, (49) of Lemma 7.3 holds true in the equivalent form:

$$
(Y-1)\left(Y-1-\frac{\lambda}{r} \pm \lambda|X|\right)>0
$$

Direct computation yields

$$
\begin{gathered}
Y-1-\frac{\lambda}{r}=-\frac{\lambda}{r} \frac{\left(r^{2}+1\right)}{\left(\lambda^{2}+1\right)^{2}} \\
|X|=\frac{\sqrt{r^{2}+1}}{r} \frac{1}{\sqrt{\lambda^{2}+1}}\left(1-\frac{\lambda^{2}\left(1+r^{2}\right)}{\left(\lambda^{2}+1\right)^{3}}\right)^{1 / 2} .
\end{gathered}
$$

Substitution in the second term of the inequality (53) yields:

$$
Y-1-\frac{\lambda}{r} \pm \lambda|X|=-\frac{\lambda}{r} \sqrt{\frac{1+r^{2}}{1+\lambda^{2}}}\left(\frac{\sqrt{r^{2}+1}}{\left(\lambda^{2}+1\right)^{3 / 2}} \mp\left(1-\frac{\lambda^{2}\left(1+r^{2}\right)}{\left(\lambda^{2}+1\right)^{3}}\right)^{1 / 2}\right)
$$

Firstly, consider the case: $Y-1-\frac{\lambda}{r}-\lambda|X|$. Substitution into (53) is equivalent to the condition

$$
-(Y-1) \lambda>0
$$


that is,

$$
1-\frac{r^{2}+1}{\left(\lambda^{2}+1\right)^{2}}<0
$$

which equivalently reads

$$
|\lambda|<\lambda_{c}=\sqrt{-1+\sqrt{r^{2}+1}}
$$

As the right hand side of (5-a) vanishes if and only if $\lambda=0$ or $\lambda= \pm \lambda_{c}$, the solution (5) of the linear system actually reduces to $\lambda \in I_{2} \cup I_{3}$. Moreover, direct computation shows that the resulting $(X= \pm L, Y-1=0, f=0)$ is a solution of (32) as well, while $\left(Y-1=0, \lambda= \pm \lambda_{c}\right)$ yields no solution.

Secondly, the case: $Y-1-\frac{\lambda}{r}+\lambda|X|$ yields no extra constraint. Indeed, the identity (54) is of the form

$$
Y-1-\frac{\lambda}{r}+|X|=C(Y-1)
$$

where $C$ is some positive constant depending on $\lambda$ and $r$. Therefore, the second case equivalently reads

$$
(Y-1)^{2}>0
$$

which is automatically realised by definition of a linear system.

This achieves the proof.

\subsection{Proof of Proposition 4.2}

Proof. The expression of $X(5-\mathrm{b})$ is equivalent to :

$$
\begin{aligned}
X^{ \pm} & = \pm \frac{1+r^{2}}{r} \sqrt{v \theta(v)}, \\
\text { with } \quad \theta(v) & \equiv v^{3}-v^{2}+\frac{1}{r^{2}+1}
\end{aligned}
$$

and where $\theta$ must be positive. Moreover, taking into account the symmetry, we focus on $\lambda>0$. The discriminant of the cubic polynomial $\theta$ is such that

$$
\Delta_{r s}=\frac{1}{\left(1+r^{2}\right)^{2}} \frac{23-4 r^{2}}{27}
$$

Hence, the critical value of the aspect ratio $r_{r s}$ is defined in (8-a). Straightforward computations shows that $\theta$ has positive roots (8) for $r \geq r_{r s}$. Furthermore, taking into account that the function $\theta$ must be positive for $\lambda \in\left[0, \lambda_{c}[\right.$ for the most, the proof is achieved because $Y$ does not induce any constraint as detailed in the following paragraph.

From (5)-a, we deduce that

$$
\begin{array}{r}
Y^{\prime}(f)=\frac{\sqrt{1+r^{2}}}{2 r} \frac{F(u)}{u^{3}} \\
F(u) \equiv u^{3}+3\left(r^{2}+1\right) u-4\left(r^{2}+1\right)
\end{array}
$$


where $u=\lambda^{2}+1$. According to Proposition 4.2, the definition range of $u$ is, for the most, such that

$$
u \in\left[1, u_{c}\left[\quad \text { with } \quad u_{c}=\sqrt{1+r^{2}} .\right.\right.
$$

The polynomial equation $\mathrm{F}$ has one real root $u_{m}$ such that

$$
u_{m}=\left(r^{2}+1\right)^{1 / 3}\left[\left(\sqrt{r^{2}+5}+2\right)^{1 / 3}-\left(\sqrt{r^{2}-5}-2\right)^{1 / 3}\right] .
$$

As $\hat{Y}$ is an odd function with respect to $\lambda$, we consider $\lambda \in\left[0, \lambda_{c}\left[\right.\right.$, that is $f \in\left[0, f_{c}[\right.$ with:

$$
f_{c}=\frac{2}{\sqrt{r^{2}+1}} \sqrt{-1+\sqrt{r^{2}+1}}
$$

Let us consider the example $r=1$. The analytic expressions of the critical values are such that

$$
\left(f_{m}=0.46, Y_{m}=0.79\right), \quad\left(f_{c}=0.91, Y_{c}=1\right),
$$

as already computed (See Figure 1).

\section{Details of the description of the definition range of $f$ of the conchoidal Branches}

The graphs of $f$ and $g$ intersect at $v=v_{r s}$. Proof. The intersection is defined as the solutions of the following system

$$
\theta(v)=0 \quad \text { and } \quad \zeta(v)=0 .
$$

Taking into account that $\zeta(v)=\theta(v)+v \theta^{\prime}(v)$ and $v \neq 0$, we find that the set under consideration reduces to

$$
\theta(v)=\theta^{\prime}(v)=0
$$

which corresponds to double roots for $\theta(v)$. Previous results show that this occurs if and only if $r=r_{r s}$, that is if and only if $v=v_{r s}$ (See (8)). This achieves the proof.

There exists a mapping $v \mapsto r(v)$ such that

$$
\lim _{\substack{v \rightarrow v_{r s} \\ v>v_{r s}}} r(v)=r_{r s} \quad \text { and } \quad \lim _{\substack{v \rightarrow v_{r s} \\ v>v_{r s}}} X^{ \pm}(r(v), v)=0 .
$$

This result is based on the diagonalization argument which is proved in [21] and stated as it follows.

Let $\varepsilon, \delta>0$ and $f: \mathbb{R}^{+} \times \mathbb{R}^{+} \rightarrow \overline{\mathbb{R}}=\mathbb{R} \cup\{-\infty,+\infty\}$. Then, there exists a mapping $\varepsilon \rightarrow \delta(\varepsilon)$, such that $\lim _{\varepsilon \rightarrow 0} \delta(\varepsilon)=0$ and

$$
\limsup _{\varepsilon \rightarrow 0} f(\delta(\varepsilon), \varepsilon) \leq \limsup _{\delta \rightarrow 0} \limsup _{\varepsilon \rightarrow 0} f(\delta, \varepsilon) .
$$


As $\partial_{\lambda} X^{ \pm}$is of class $\mathcal{C}^{0}$ in some neighbourhood of $\left(r_{r s}, v_{r s}\right)$ and the roots $v_{p}$ are continuous functions of the parameter $r$, we have :

$$
\lim _{\substack{r \rightarrow r_{r s} \\ r<r_{r s}}} \lim _{v \rightarrow v_{p}(r)} \partial_{\lambda} X^{ \pm}(r, v)=\lim _{\substack{r \rightarrow r_{r s} \\ r<r_{r s}}} \partial_{\lambda} X^{ \pm}\left(r, v_{p}(r)\right)=0
$$

Proof. The last equality results from the fact that $v_{p}$ is a root of $\zeta$ and of (10-a). Thus, Lemma 8 yields the existence of a mapping $v \mapsto r(v)$ that satisfies (58). 